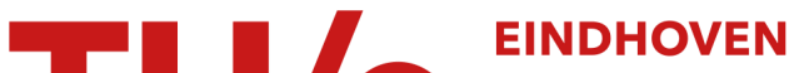 \\ UNIVERSITY OF \\ TECHNOLOGY
}

\section{Cyclic queueing networks with subexponential service times}

Citation for published version (APA):

Ayhan, H., Palmowski, Z. B., \& Schlegel, S. (2002). Cyclic queueing networks with subexponential service times. (Report Eurandom; Vol. 2002024). Eurandom.

Document status and date:

Published: 01/01/2002

\section{Document Version:}

Publisher's PDF, also known as Version of Record (includes final page, issue and volume numbers)

\section{Please check the document version of this publication:}

- A submitted manuscript is the version of the article upon submission and before peer-review. There can be important differences between the submitted version and the official published version of record. People interested in the research are advised to contact the author for the final version of the publication, or visit the $\mathrm{DOI}$ to the publisher's website.

- The final author version and the galley proof are versions of the publication after peer review.

- The final published version features the final layout of the paper including the volume, issue and page numbers.

Link to publication

\section{General rights}

Copyright and moral rights for the publications made accessible in the public portal are retained by the authors and/or other copyright owners and it is a condition of accessing publications that users recognise and abide by the legal requirements associated with these rights.

- Users may download and print one copy of any publication from the public portal for the purpose of private study or research.

- You may not further distribute the material or use it for any profit-making activity or commercial gain

- You may freely distribute the URL identifying the publication in the public portal.

If the publication is distributed under the terms of Article $25 \mathrm{fa}$ of the Dutch Copyright Act, indicated by the "Taverne" license above, please follow below link for the End User Agreement:

www.tue.nl/taverne

\section{Take down policy}

If you believe that this document breaches copyright please contact us at:

openaccess@tue.nl

providing details and we will investigate your claim. 


\title{
Cyclic Queueing Networks with Subexponential Service Times
}

\author{
H. Ayhan ${ }^{1,4}$ \\ Z. Palmowski ${ }^{2,3,5}$ \\ S. Schlegel ${ }^{2}$
}

May 17, 2002

\begin{abstract}
For a $K$-stage cyclic queueing model with $N$ customers and general service times we give an explicit expression for the $n^{\text {th }}$ departure time from each stage. Starting from this expression we analyze the asymptotic tail behaviour of cycle times and waiting times given that at least one service time distribution is subexponential. Furthermore, we show that the tail of the residual of a subexponential service time seen by an arriving customer is of the same order as the service time itself where the asymptotic constant depends on the queue length on arrival.
\end{abstract}

KEyWORds: CYCLE TIME, CLOSED NETWORK, SUBEXPONENTIAL ASYMPTOTICS, HARRIS MARKOV CHAIN

2000 Mathematics Subject Classification: 60K25, 90B22, 60K20

\section{Introduction}

We consider a cyclic $K$-stage $(K \geq 2)$ queueing system as shown in Figure 1. There is a single server at station $i(i=1, \ldots, K)$ and the service discipline at all stations is First Come First

\footnotetext{
${ }^{1}$ School of Industrial and Systems Engineering, Georgia Institute of Technology, Atlanta, GA 30332-0205, USA

${ }^{2}$ EURANDOM, P.O. Box 513, 5600 MB Eindhoven, The Netherlands

${ }^{3}$ Mathematical Institute, University of Wrocław, pl. Grunwaldzki 2/4, 50-384 Wrocław, Poland

${ }^{4}$ Research was supported by the National Science Foundation under Grants DMI-9908161, and DMI-9984352

${ }^{5}$ Research was supported by KBN under grant 5 P03A 02120
} 


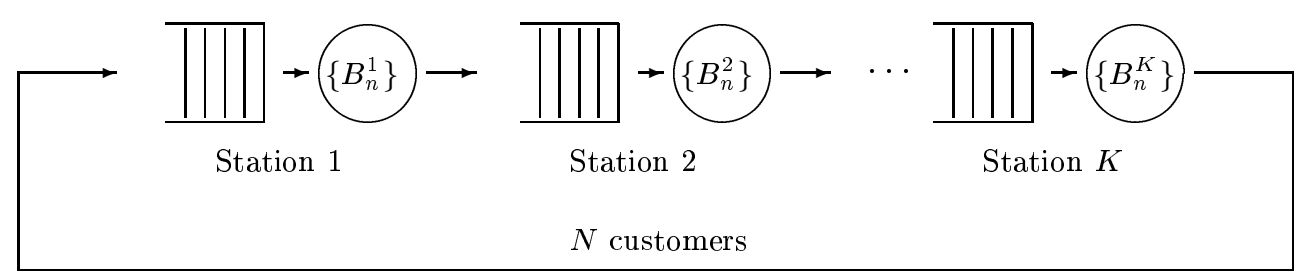

Figure 1: Cyclic queueing network $K$ queues

Served (FCFS). The capacity of the buffer between two consecutive stations is infinite. There are $N$ customers in the system, who cyclically visit station 1 to station $K$. We assume that at time zero there are $N_{i}$ customers in front of station $i, i=1, \ldots, K$. Hence, $\sum_{i=1}^{K} N_{i}=N$. Service times at station $i$ are independent and identically distributed random variables $\left\{B_{n}^{i}\right\}$ with distribution function $B_{i}(\cdot)$. and the sequences $\left\{B_{n}^{1}\right\}, \ldots,\left\{B_{n}^{K}\right\}$ are assumed to be mutually independent. Furthermore, we assume that there exists a subexponential distribution $F(\cdot)(F \in \mathcal{S})$ and there exist constants $c_{i} \in[0, \infty)$ with $\sum_{i=1}^{K} c_{i}>0$ such that for all $i=1, \ldots, K$

$$
\lim _{x \rightarrow \infty} \frac{\bar{B}_{i}(x)}{\bar{F}(x)}=c_{i}
$$

The tail behaviour of the cycle time (the time between successive departures of the same customer from a given station $i, i=1, \ldots, K)$, waiting time at station $i(i=1, \ldots, K)$ and residual service time at station $i(i=1, \ldots, K)$ seen by an arriving customer are main objects of our interest. Relatively few mathematical methods are available for treating these quantities in closed queueing networks. The reason for this is that a customer passing through the system experiences the whole space-time structure of the network state process. One can use general methods for closed Gordon-Newell networks [18], Laplace transform techniques [8, 11, 24], reversibility arguments [12], standard embedded Markov chain techniques [15], or the theory of point processes [16]. All of these results are valid under the assumption that service times are exponential random variables. Boxma [8] derives an expression for the Laplace-Stieltjes transform of the distribution of the stationary cycle time in a two-stage cyclic queueing network with one exponential and one general station. This result reveals a surprising phenomenon: in general, the distribution of the cycle time 
depends on the order in which two stations are visited. As Boxma [8] noted, this is because of the dependence between successive response times at two queues. As far as the open networks with subexponential service times are concerned, Baccelli, Schlegel and Schmidt [4] consider the tail behaviour of stationary response times in open $(\max ,+)$ linear systems. In a similar paper, Huang and Sigman [19] focus on the asymptotics of sojourn times and queue lengths in tandem queues and split-match queues. More recently Baccelli and Foss [5] compute upper and lower bounds for the tail asymptotics of the stationary maximal dater in more general monotone-separable stochastic networks. Moreover, they obtain exact asymptotics for various special cases of these networks.

In this paper we provide an explicit representation for the $n^{\text {th }}$ departure epoch from any given station (for similar results in open networks see e.g. [4]). This in turn allows us to obtain the subexponential asymptotics of the $n^{\text {th }}$ cycle time at station $i$ which is denoted by $C_{n}^{i}$. In particular, we have

$$
\mathbb{P}\left(C_{n}^{i}>x\right) \sim N \sum_{\ell=1}^{K} c_{\ell} \bar{F}(x) \quad \text { as } x \rightarrow \infty, \forall n \geq N
$$

where $f(x) \sim g(x)$ as $x \rightarrow \infty$ means that $\lim _{x \rightarrow \infty} f(x) / g(x)=1$ for two functions $f(x)$ and $g(x)$. Note that the cycle time asymptotics is the same for each station. This is different for the cycle time distribution, which indeed depends on the station where the cycle starts; see [8]. Next we study the tail behaviour of the distribution of the $n^{\text {th }}$ waiting time at station $i$ for $i=1, \ldots, K$. In order to give an intuitive explanation for the behaviour of the network, we also analyze the tail behaviour of the distribution of a residual service seen by an arriving customer given that he sees $k$ customers in front of him. We show that this tail is of the same order as the tail of the service time distribution at the corresponding station. This is in contrast to open systems where the residual service time distribution is "heavier" than the service time distribution itself (see for example [20]). Using Harris recurrence methods we prove that if the service times at each station have infinite support, then there exists a random variable $C^{i}$ such that

$$
C_{n}^{i} \stackrel{\mathcal{D}}{\rightarrow} C^{i}
$$

holds as $n \rightarrow \infty$ where $\stackrel{\mathcal{D}}{\rightarrow}$ denotes convergence in distribution. The sequence $C_{n}^{i}$ couples with $C^{i}$ in finite time for all initial conditions $N_{1}, N_{2}, \ldots, N_{K}$ such that $\sum_{i=1}^{K} N_{i}=N$. Using (1) and (2) 
we obtain the subexponential asymptotics for $C^{i}$ :

$$
\mathbb{P}\left(C^{i}>x\right) \sim N \sum_{\ell=1}^{K} c_{\ell} \bar{F}(x) \quad \text { as } x \rightarrow \infty .
$$

Results similar to the one in (3) are also obtained for stationary waiting times and stationary residual service times. To the best of our knowledge, our results are the first of this kind for cyclic queues with more than two stations and non-exponential service times.

The paper is organized as follows. In Section 2, in order to provide the reader with a better understanding of our results we consider the two stage cyclic queueing network. In Section 3, we derive an explicit expression for the $n^{\text {th }}$ departure time from station $i$. We use this result in Section 4 to get subexponential asymptotics for the $n^{\text {th }}$ cycle time $C_{n}^{i}$. In Section 5 , we obtain asymptotics for the $n^{\text {th }}$ waiting time at station $i$. Asymptotic tail behaviour of the $n^{\text {th }}$ residual service time at station $i$ is considered in Section 6 . In Section 7, we prove (3) and also obtain the results on tail behaviour of stationary waiting times and stationary residual service times. Finally, in the appendix we first present the proof of a technical lemma and then recall some properties of subexponential distributions used in this paper.

\section{Two-stage Cyclic Queue}

In this section, we analyze the case of two stations and two customers (i.e. $K=2$ and $N=2$ ). In particular we consider the $n^{\text {th }}$ cycle time at station $i$ which is the time between the $(n+N)^{\text {th }}$ and $n^{\text {th }}$ departures from station $i$. Recall that $C_{n}^{i}$ denotes the $n^{\text {th }}$ cycle time for station $i$ and let $X_{n}^{i}$ denote the $n^{\text {th }}$ departure time from station $i(i=1,2)$.

The location of the customers in the network at time 0 is called the initial marking. We will see that the stationary regime does not depend on this initial marking. In fact, the influence of the initial marking already vanishes after two iterations when $K=2$ and $N=2$. Without loss of generality we assume that no customers are being served at time 0 . Throughout the paper we use the following notation

$$
[j]= \begin{cases}K & \text { if } j \bmod K=0 \\ j \bmod K & \text { if } j \bmod K \neq 0\end{cases}
$$


where $K$ is the number of stations as defined above. Furthermore, we use the symbol $\oplus$ for maximization, and the symbol $\otimes$ for addition. Thus, we write $a \oplus b$ for $\max \{a, b\}$, and $a \otimes b$ (or shortly $a b$ ) for $a+b$ where $a$ and $b$ are real numbers. This is the standard $(\max ,+$ ) algebra notation (see [3] for more details on this formalism). Note that as in conventional algebra $\otimes$ has precedence over $\oplus$. Even though cyclic tandem queue is an example of a $(\max ,+)$ linear system, general $(\max ,+)$ linear systems and $(\max ,+)$ algebra are beyond the scope of this paper. We use these symbols simply for notational convenience. Without loss of generality we set

$$
X_{0}^{i}=0
$$

One can easily see that in any cyclic queue for all $1=1, \ldots, K$

$$
X_{n}^{i}=B_{n}^{i} X_{n-1}^{i} \oplus B_{n}^{i} X_{n-N_{i}}^{[i-1]}
$$

For the tandem queue with two stations and two customers, we focus on $C_{n}^{1}$. We first consider the case that initially there is one customer at each station (i.e., $N_{1}=N_{2}=1$ ). Then,

$$
X_{1}^{1}=B_{1}^{1}, \quad X_{1}^{2}=B_{1}^{2}
$$

and

$$
\begin{aligned}
C_{1}^{1} & =X_{3}^{1}-X_{1}^{1}=B_{3}^{1}\left(B_{1}^{1} \oplus B_{1}^{2}\right)\left(B_{2}^{1} \oplus B_{2}^{2}\right)-B_{1}^{1} \\
& =\left(0 \oplus\left(B_{1}^{2}-B_{1}^{1}\right)\right)\left(B_{2}^{1} \oplus B_{2}^{2}\right) B_{3}^{1}, \\
C_{2}^{1} & =X_{4}^{1}-X_{2}^{1}=\left(B_{3}^{1} \oplus B_{3}^{2}\right)\left(B_{2}^{1} \oplus B_{2}^{2}\right) B_{4}^{1}-B_{2}^{1} \\
& =\left(0 \oplus\left(B_{2}^{2}-B_{2}^{1}\right)\right)\left(B_{3}^{1} \oplus B_{3}^{2}\right) B_{4}^{1} .
\end{aligned}
$$

In general, for all $n \geq 1$

$$
\begin{aligned}
C_{n}^{1} & =X_{n+2}^{1}-X_{n}^{1} \\
& =\left(0 \oplus\left(B_{n}^{2}-B_{n}^{1}\right)\right)\left(B_{n+1}^{1} \oplus B_{n+1}^{2}\right) B_{n+2}^{1} .
\end{aligned}
$$

Thus from Lemmas 8.4, 8.5, and 8.6 we have

$$
\mathbb{P}\left(C_{n}^{1}>x\right) \sim 2\left(c_{1}+c_{2}\right) \bar{F}(x), x \rightarrow \infty .
$$


Next, we initially place both customers at station 1 (i.e., $N_{1}=2, N_{2}=0$ ) and again consider $C_{n}^{1}$. Then,

$$
X_{1}^{1}=B_{1}^{1}, \quad X_{2}^{1}=B_{2}^{1} B_{1}^{1}, \quad X_{1}^{2}=B_{1}^{2} B_{1}^{1} .
$$

Note that at the completion of the first service time at station 1 (i.e., after $B_{1}^{1}$ has elapsed) we are in the first scenario. Thus, the $n^{\text {th }}$ cycle time for this initial marking is the same as the $(n-1)^{\text {th }}$ cycle time for the first initial marking with subscripts of $B^{1}$ increased by one. We have from (5) for all $n \geq 2$

$$
\begin{aligned}
C_{n}^{1} & =X_{n+2}^{1}-X_{n}^{1} \\
& =\left(0 \oplus\left(B_{n-1}^{2}-B_{n}^{1}\right)\right)\left(B_{n}^{2} \oplus B_{n+1}^{1}\right) B_{n+2}^{1},
\end{aligned}
$$

which again gives (6). Finally, we consider the case that both customers are at station 2 at time 0 (i.e., $N_{1}=0, N_{2}=2$ ). At the completion of the first service time at station 1 (i.e. after $B_{1}^{2}$ has elapsed) we are in the first scenario. Thus, the $n^{\text {th }}$ cycle time for this initial marking is the same as the $n^{\text {th }}$ cycle time of the first initial marking but now with subscripts of $B^{2}$ increased by one.

We have for all $n \geq 1$

$$
\begin{aligned}
C_{n}^{1} & =X_{n+2}^{1}-X_{n}^{1} \\
& =\left(0 \oplus\left(B_{n+1}^{2}-B_{n}^{1}\right)\right)\left(B_{n+1}^{1} \oplus B_{n+2}^{2}\right) B_{n+2}^{1}
\end{aligned}
$$

and therefore again (6). So for the case of two queues and two customers, $C_{n}^{i}$ does not depend on the initial marking for all $n \geq 2$. This follows since after at most one initial service (namely in the case that both customers are at the same station at time 0 ), services at station 1 and station 2 always start at the same time.

\section{Departure Times}

In this section we derive an explicit expression for the $n^{\text {th }}$ departure time from station $i, i=$ $1, \ldots, K$. Throughout our developments we set $B_{j}^{k}=0$ for $j \leq 0$. Let $\lfloor u\rfloor$ denote the largest integer less than or equal to $u$ and let $\lceil u\rceil$ denote the smallest integer greater than or equal to $u$. 
Proposition 3.1 The $n^{\text {th }}$ departure time $X_{n}^{i}$ from station $i, i=1, \ldots, K$, is given by

$$
\begin{aligned}
X_{n}^{i}= & \bigotimes_{k=1}^{n} B_{k}^{i} \oplus \bigoplus_{j=1}^{\infty} \bigoplus_{j_{1}=N j-\left(\sum_{k=1}^{K-1} N_{[i-k]}-1\right)}^{n} \bigoplus_{r=2}^{K j} \bigoplus_{j_{r}=N j-(f(r)-1)}^{j_{r-1}-N_{[i-r+2]}} \\
& \left(\bigotimes_{i_{1}=j_{1}}^{n} B_{i_{1}}^{i} \bigotimes_{q=2}^{K j} \bigotimes_{i_{q}=j_{q}}^{j_{q-1}-N_{[i-q+2]}} B_{i_{q}}^{[i-q+1]} \bigotimes_{l=1}^{j_{K j}-N_{[i-K+1]}} B_{l}^{i}\right)
\end{aligned}
$$

where

$$
f(r)= \begin{cases}\left\lfloor\frac{r}{K}\right\rfloor N_{i}+\left\lceil\frac{r}{K}\right\rceil \sum_{k=1}^{K-1} N_{[i-k]} & \text { for }[r]=1 \\ \left\lceil\frac{r}{K}\right\rceil \sum_{k=[r]-1}^{K} N_{[i-k]}+\left(\left\lceil\frac{r}{K}\right\rceil+1\right) \sum_{k=1}^{[r]-2} N_{[i-k]} & \text { for }[r]=2, \ldots, K,\end{cases}
$$

with the convention that $\oplus$ over an empty set is $-\infty$ and $\otimes$ over an empty set is 0 .

More explicitly (7) can be written as

$$
\begin{aligned}
& X_{n}^{i}=\bigotimes_{k=1}^{n} B_{k}^{i} \oplus \bigoplus_{j=1}^{\infty} \underset{j_{1}=N j-\left(\sum_{k=1}^{K-1} N_{[i-k]}-1\right)}{n} \stackrel{\left.\bigoplus_{2}=N j-\left(\sum_{k=1}^{K} \underline{N}_{[i}-k\right]-1\right)}{j_{1}-N_{i}} \\
& j_{2}-N_{[i-1]} \quad j_{3}-N_{[i-2]} \\
& \bigoplus \bigoplus
\end{aligned}
$$

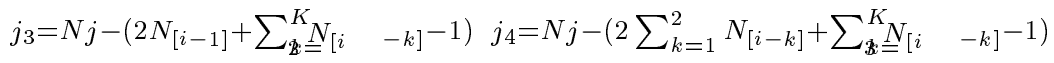

$$
\begin{aligned}
& j_{K+1}-N_{[i-K]} \quad j_{K+2}-N_{[i-1]} \\
& \cdots \bigoplus_{j_{K+2}=N j-\left(2 \sum_{k=1}^{K} N_{[i-k]}-1\right)} \bigoplus_{j_{K+3}=N j-\left(3 N_{[i-1]}+2 \sum_{k=2}^{K} N_{[i-k]}-1\right)}^{\cdots} \ldots \\
& \cdots \bigoplus_{j_{2 K+2}=N j-\left(3 \sum_{k=1}^{K} N_{[i-k]}-1\right)}^{j_{2 K+1}-N_{[i-K]}} \cdots \bigoplus_{j_{K j}=1}^{j_{K j-1}-N_{[i-(K-2)]}}\left(\bigotimes_{i_{1}=j_{1}}^{n} B_{i_{1}}^{i} \bigotimes_{i_{2}=j_{2}}^{j_{1}-N_{i}} B_{i_{2}}^{[i-1]}\right. \\
& \bigotimes_{i_{3}=j_{3}}^{j_{2}-N_{[i-1]}} B_{i_{3}}^{[i-2]} \bigotimes_{i_{4}=j_{4}}^{j_{3}-N_{[i-2]}} B_{i_{4}}^{[i-3]} \cdots \bigotimes_{i_{K}=j_{K}}^{j_{K-1}-N_{[i-(K-2)]}} B_{i_{K}}^{[i-K+1]} \\
& \left.\bigotimes_{i_{K+1}=j_{K+1}}^{j_{K}-N_{[i-(K-1)]}} B_{i_{K+1}}^{i} \ldots \ldots \bigotimes_{i_{K j}=j_{K j}}^{j_{K j-1}-N_{[i-(K-2)]}} B_{i_{K j}}^{[i-K+1]} \bigotimes_{l=1}^{j_{K j}-N_{[i-(K-1)]}} B_{l}^{i}\right) .
\end{aligned}
$$

We will use (8) in our developments since this more explicit representation will provide the reader with more illustrative proofs that are not difficult to follow. Before giving the proof of Proposition 3.1, we present a lemma which provides an equivalent representation for departure times. The proof is given in the appendix.

Lemma 3.1 The expression for departure times given in (7) is equivalent to

$$
X_{n}^{i}=\bigoplus_{j=1}^{\infty} \bigoplus_{j_{1}=N j-\left(\sum_{k=1}^{K} N_{[i-k]}-1\right)}^{n} \bigoplus_{r=2}^{K j-1} \bigoplus_{j_{r}=N j-(g(r)-1)}^{j_{r-1}-N_{[i-r+2]}}
$$




$$
\left(\bigotimes_{i_{1}=j_{1}}^{n} B_{i_{1}}^{i} \bigotimes_{q=2}^{K j-1} \bigotimes_{i_{q}=j_{q}}^{j_{q-1}-N_{[i-q+2]}} B_{i_{q}}^{[i-q+1]} \bigotimes_{l=1}^{j_{K j-1}-N_{[i-K+2]}} B_{l}^{[i-K+1]}\right)
$$

where

$$
g(r)= \begin{cases}\left\lceil\frac{r}{K}\right\rceil \sum_{k=1}^{K} N_{[i-k]} & \text { for }[r]=1 \\ \left\lceil\frac{r}{K}\right\rceil \sum_{k=[r]-1}^{K-1} N_{[i-k]}+\left(\left\lceil\frac{r}{K}\right\rceil+1\right)\left(\sum_{k=0}^{[r]-2} N_{[i-k]}\right) & \text { for }[r]=2, \cdots, K .\end{cases}
$$

Proof of Proposition 3.1

We use induction. Without loss of generality we assume that $X_{n}^{i}=0$, for all $n \leq 0$ and $i=1, \ldots, K$.

We first show that (8) is correct for $n=1$. First let $i$ be a station such that $N_{i}>0$. Such an $i$ always exists since $N>0$. In this case, (8) reduces to

$$
X_{1}^{i}=\bigotimes_{k=1}^{1} B_{k}^{i}=B_{1}^{i}
$$

On the other hand, it follows from (4) and our assumptions on initial conditions that $X_{1}^{i}=B_{1}^{i}$.

Next assume $i$ is such that $N_{i}=0$ and $N_{[i-1]}>0$. Then (8) reduces to

$$
X_{1}^{i}=\bigotimes_{k=1}^{1} B_{k}^{i} \oplus \bigoplus_{j_{1}=1}^{1} \bigoplus_{j_{2}=1}^{j_{1}}\left(\bigotimes_{i_{1}=j_{1}}^{1} B_{i_{1}}^{i} \bigotimes_{i_{2}=j_{2}}^{j_{1}} B_{i_{2}}^{[i-1]}\right)=B_{1}^{i} B_{1}^{[i-1]} \text {. }
$$

Similarly, from (4), our assumptions on initial conditions and the fact that $X_{1}^{[i-1]}=B_{1}^{[i-1]}$ (which follows from (10)) we have

$$
X_{1}^{i}=B_{1}^{i} \oplus B_{1}^{i} B_{1}^{[i-1]}=B_{1}^{i} B_{1}^{[i-1]}
$$

Continuing in this fashion, from (4) we have

$$
X_{1}^{i}=B_{1}^{i} B_{1}^{[i-1]} B_{1}^{[i-2]} \cdots B_{1}^{[i-k]}
$$

for $i$ with $N_{i}=N_{[i-1]}=\ldots=N_{[i-(k-1)]}=0$ and $N_{[i-k]}>0$, and any $k=1, \ldots, K-1$. In this case (8) also reduces to

$$
\begin{aligned}
X_{1}^{i} & =\bigotimes_{k=1}^{1} B_{k}^{i} \oplus \bigoplus_{j_{1}=1}^{1} \bigoplus_{j_{2}=1}^{j_{1}} \cdots \bigoplus_{j_{k+1}=1}^{j_{k}}\left(\bigotimes_{i_{1}=j_{1}}^{1} B_{i_{1}}^{i} \bigotimes_{i_{2}=j_{2}}^{j_{1}} B_{i_{2}}^{[i-1]} \cdots \bigotimes_{i_{k+1}=j_{k+1}}^{j_{k}} B_{i_{k+1}}^{[i-k]}\right) \\
& =B_{1}^{i} B_{1}^{[i-1]} \cdots B_{1}^{[i-k]}
\end{aligned}
$$

which completes the proof for $n=1$. Now suppose that (8) holds for $m \leq n-1$. Thus, it follows from (9) that for all $m \leq n-1$

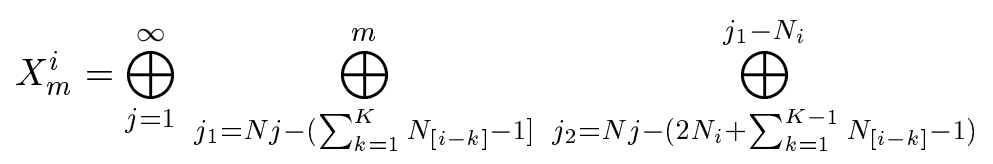




$$
\begin{aligned}
& j_{2}-N_{[i-1]} \\
& \underset{j_{3}=N j-\left(2 N_{i}+2 N_{[i-1]}+\sum_{k=2}^{K-1} N_{[i-k]}-1\right)}{j_{K+1}-N_{i}} \stackrel{j_{4}=N j-\left(2 N_{i}+2 \sum_{k=1}^{2} N_{[i-k]}+\sum_{k=3}^{K-1} N_{[i-k]}-1\right)}{j_{K+2}-N_{[i-1]}} \\
& j_{3}-N_{[i-2]} \\
& \cdots \bigoplus_{j_{K+2}=N j-\left(3 N_{i}+2 \sum_{k=1}^{K-1} N_{[i-k]}-1\right)}^{j_{j}+3=N j-\left(3 N_{i}+3 N_{[i-1]}+2 \sum_{k=2}^{K-1} N_{[i-k]}-1\right)} \cdots \\
& \ldots \bigoplus_{j_{2 K+2}=N j-\left(4 N_{i}+3 \sum_{k=1}^{K-1} N_{[i-k]}-1\right)}^{j_{2 K+1}-N_{i}} \cdots \bigoplus_{j_{K j-1}=1}^{j_{K j-2}-N_{[i+3]}}\left(\bigotimes_{i_{1}=j_{1}}^{m} B_{i_{1}}^{i} \bigotimes_{i_{2}=j_{2}}^{j_{1}-N_{i}} B_{i_{2}}^{[i-1]}\right. \\
& \bigotimes_{i_{3}=j_{3}}^{j_{2}-N_{[i-1]}} B_{i_{3}}^{[i-2]} \bigotimes_{i_{4}=j_{4}}^{j_{3}-N_{[i-2]}} B_{i_{4}}^{[i-3]} \cdots \bigotimes_{i_{K}=j_{K}}^{j_{K}-1-N_{[i+2]}} B_{i_{K}}^{[i+1]} \\
& \left.\bigotimes_{i_{K+1}=j_{K+1}}^{j_{K}-N_{[i+1]}} B_{i_{K+1}}^{i} \cdots \bigotimes_{i_{K j-1}=j_{K j-1}}^{j_{K j-2}-N_{[i+3]}} B_{i_{K j-1}}^{[i+2]} \bigotimes_{l=1}^{j_{K j-1}-N_{[i+2]}} B_{l}^{[i+1]}\right) .
\end{aligned}
$$

First assume that $i$ is such that $N_{i}>0$. Then we have

$$
\begin{aligned}
& X_{n}^{i}=\left(\bigotimes_{k=1}^{n} B_{k}^{i} \oplus \bigoplus_{j=1}^{\infty} \bigoplus_{j_{1}=N j-\left(\sum_{k=1}^{K-1} N_{[i-k]}-1\right)}^{n-1} \stackrel{\left.j_{2}=N j-\left(\sum_{k=\underline{N_{[i}}}^{K_{1}}-k\right]-1\right)}{j_{1}-N_{i}}\right. \\
& j_{2}-N_{[i-1]} \quad j_{3}-N_{[i-2]} \\
& \bigoplus \\
& j_{3}=N j-\left(2 N_{[i-1]}+\sum_{k=2}^{K} N_{[i-k]}-1\right) \quad j_{4}=N j-\left(2 \sum_{k=1}^{2} N_{[i-k]}+\sum_{k=3}^{K} N_{[i-k]}-1\right) \\
& j_{K+1}-N_{i} \quad j_{K+2}-N_{[i-1]}
\end{aligned}
$$

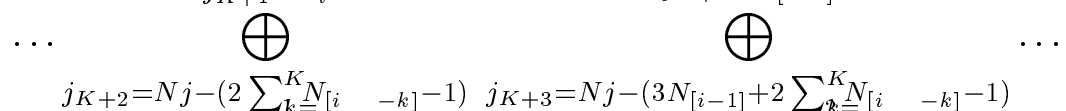

$$
\begin{aligned}
& j_{2 K+1}-N_{i} \quad j_{K j-1}-N_{[i+2]} n^{j_{K}} j_{1}-N_{i} \\
& \cdots \bigoplus_{\left.j_{2 K+2}=N j-\left(3 \sum_{k}^{K_{N} N_{i i}}-k\right]-1\right)}^{j_{2 K+1}-N_{i}} \cdots \bigoplus_{j_{K j}=1}^{j_{K j-1}-N_{[i+2]}}\left(\bigotimes_{i_{1}=j_{1}}^{n} B_{i_{1}}^{i} \bigotimes_{i_{2}=j_{2}}^{j_{1}-N_{i}} B_{i_{2}}^{[i-1]}\right. \\
& \bigotimes_{i_{3}=j_{3}}^{j_{2}-N_{[i-1]}} B_{i_{3}}^{[i-2]} \bigotimes_{i_{4}=j_{4}}^{j_{3}-N_{[i-2]}} B_{i_{4}}^{[i-3]} \cdots \bigotimes_{i_{K}=j_{K}}^{j_{K-1}-N_{[i+2]}} B_{i_{K}}^{[i+1]} \\
& j_{K}-N_{[i+1]} \quad j_{K j-1}-N_{[i+2]} \quad j_{K j}-N_{[i+1]} \\
& \left.\left.\bigotimes_{i_{K+1}=j_{K+1}}^{j_{K}} B_{i_{K+1}}^{i} \ldots \bigotimes_{i_{K j}=j_{K j}}^{[i+2]} B_{i_{K j}}^{[i+1]} \bigotimes_{l=1}^{j_{K j}} B_{l}^{i}\right)\right) \oplus
\end{aligned}
$$

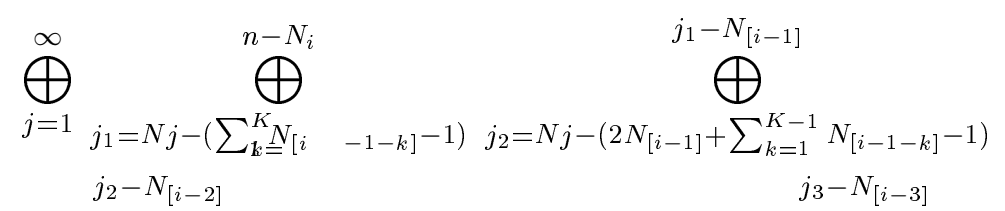

$$
\begin{aligned}
& \bigoplus \bigoplus \bigoplus \\
& j_{3}=N j-\left(2 N_{[i-1]}+2 N_{[i-2]}+\sum_{k=2}^{K-1} N_{[i-1-k]}-1\right) \quad j_{4}=N j-\left(2 N_{[i-1]}+2 \sum_{k=1}^{2} N_{[i-1-k]}+\sum_{k=3}^{K-1} N_{[i-1-k]}-1\right) \\
& j_{K+1}-N_{[i-1]} \quad j_{K+2}-N_{[i-2]} \\
& \cdots \bigoplus_{j_{K+2}=N j-\left(3 N_{[i-1]}+2 \sum_{k=1}^{K-1} N_{[i-1-k]}-1\right)} \bigoplus_{j_{K+3}=N j-\left(3 N_{[i-1]}+3 N_{[i-2]}+2 \sum_{k=2}^{K-1} N_{[i-1-k]}-1\right)} .
\end{aligned}
$$




$$
\begin{aligned}
& j_{2 K+1}-N_{[i-1]} \\
& \bigoplus \\
& \bigoplus_{j_{K j}-1}^{j_{K j-2}-N_{[i+2]}}\left(B_{n}^{i} \bigotimes_{i_{1}=j_{1}}^{n-N_{i}} B_{i_{1}}^{[i-1]} \bigotimes_{i_{2}=j_{2}}^{j_{1}-N_{[i-1]}} B_{i_{2}}^{[i-2]}\right. \\
& j_{2 K+2}=N j-\left(4 N_{[i-1]}+3 \sum_{k=1}^{K-1} N_{[i-1-k]}-1\right) \quad j_{K j-1}=1 \\
& \bigotimes_{i_{3}=j_{3}}^{j_{2}-N_{[i-2]}} B_{i_{3}}^{[i-3]} \bigotimes_{i_{4}=j_{4}}^{j_{3}-N_{[i-3]}} B_{i_{4}}^{[i-4]} \cdots \bigotimes_{i_{K}=j_{K}}^{j_{K-1}-N_{[i+1]}} B_{i_{K}}^{i} \\
& \left.\bigotimes_{i_{K+1}=j_{K+1}}^{j_{K}-N_{i}} B_{i_{K+1}}^{[i-1]} \cdots \bigotimes_{i_{K j-1}=j_{K j-1}}^{j_{K j-2}-N_{[i+2]}} B_{i_{K j-1}}^{[i+1]} \bigotimes_{l=1}^{j_{K j-1}-N_{[i+1]}} B_{l}^{i}\right) \\
& =\left(\bigotimes_{k=1}^{n} B_{k}^{i} \oplus \bigoplus_{j=1}^{\infty} \bigoplus_{j_{1}=N j-\left(\sum_{k=1}^{K-1} N_{[i-k]}-1\right)}^{n-1} \bigoplus_{j_{2}=N j-\left(\sum_{j_{2}-N_{[i-1]}^{K}}^{j_{1}-N_{i}} \bigoplus_{3}-N_{[i-2}^{k}\right]}^{\left.\left.N_{[i}-k\right]-1\right)}\right. \\
& \oplus \\
& \oplus \\
& j_{3}=N j-\left(2 N_{[i-1]}+\sum_{j_{K+1}-\mathcal{K}_{F_{i}}{ }^{2}}^{K} N_{[i}{ }^{-k]-1)} j_{4}=N j-\left(2 \sum_{j k=1}^{2} N_{\left[j_{[i-1]}\right.}{ }^{-k]}+\sum_{k=3}^{K} N_{[i}{ }^{-k]-1)}\right.\right. \\
& \cdots \bigoplus_{j_{K+2}=N j-\left(2 \sum^{K}\right.} \bigoplus_{-k]-1)}
\end{aligned}
$$

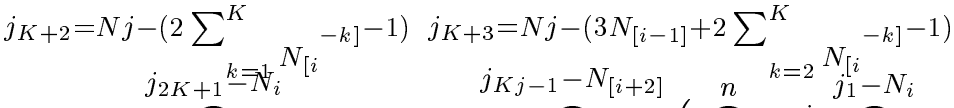

$$
\begin{aligned}
& \cdots \bigoplus_{j_{2 K+2}=N j-\left(3 \sum^{K}{ }_{-k]}-1\right)}^{j_{2 K}+1-N_{i}} \cdots \bigoplus_{j_{K j}=1}^{j_{K j}-1-N_{[i+2]}}\left(\bigotimes_{i_{1}=j_{1}}^{n} B_{i_{1}}^{i} \bigotimes_{i_{2}=j_{2}}^{j_{1}-N_{i}} B_{i_{2}}^{[i-1]}\right. \\
& \bigotimes_{i_{3}=j_{3}}^{j_{2}-N_{[i-1]}} B_{i_{3}}^{[i-2]} \bigotimes_{i_{4}=j_{4}}^{\left.j_{3}-h \bar{F}_{[i]}^{1}-2\right]} B_{i_{4}}^{N_{[i}} \ldots \bigotimes_{i_{K}=j_{K}}^{[i-3]} B_{i_{K}}^{[i+1]} \\
& \left.\left.\bigotimes_{i_{K+1}=j_{K+1}}^{j_{K}-N_{[i+1]}} B_{i_{K+1}}^{i} \ldots \ldots \bigotimes_{i_{K j}=j_{K j}}^{j_{K j-1}-N_{[i+2]}} B_{i_{K j}}^{[i+1]} \bigotimes_{l=1}^{j_{K j}-N_{[i+1]}} B_{l}^{i}\right)\right) \oplus
\end{aligned}
$$

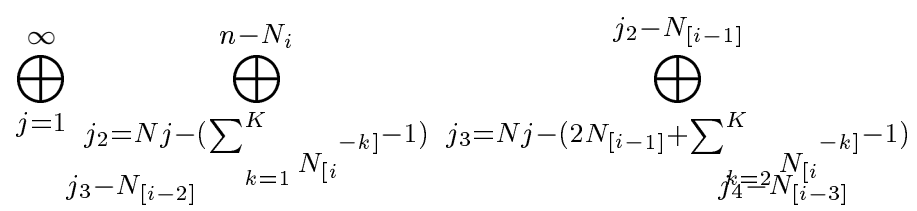

$$
\begin{aligned}
& \oplus
\end{aligned}
$$

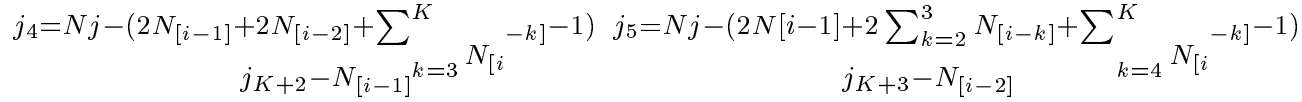

$$
\begin{aligned}
& \text { ․ } \bigoplus^{j_{K}} \bigoplus^{i-2]} \quad \ldots
\end{aligned}
$$

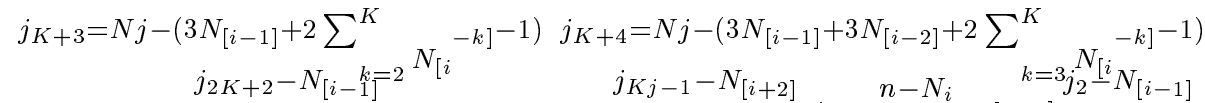

$$
\begin{aligned}
& \cdots \bigoplus_{j_{2 K+3}=N j-\left(4 N_{[i-1]}+3 \sum^{K}{ }_{-k]}-1\right)}^{\cdots} \bigoplus_{j_{K j}=1}^{j_{K j-1}-N_{[i+2]}}\left(B_{n}^{i} \bigotimes_{i_{2}=j_{2}}^{n-N_{i}} B_{i_{2}}^{[i-1]} \bigoplus_{i_{3}=j_{3}}^{k^{k}=j_{2}-N_{[i-1]}} B_{i_{3}}^{[i-2]}\right. \\
& \begin{array}{ccc}
j_{2 K+3}= & N j-\left(4 N_{[i-1]}+3 \sum^{K}{ }_{{ }^{2}} N_{\bar{j}_{4}}-N_{[i-3]}^{-k]}-1\right) & j_{K j}=1 \\
j_{3}-N_{[i-2]} & j_{K}-N_{[i+1]}
\end{array} \\
& \bigotimes_{i_{4}=j_{4}} B_{i_{4}}^{[i-3]} \bigotimes_{i_{5}=j_{5}}^{[i-3]} B_{i_{5}}^{[i-4]} \cdots \bigotimes_{i_{K+1}=j_{K+1}}^{B_{i_{K+1}}^{i}}
\end{aligned}
$$




$$
\begin{aligned}
& \left.\bigotimes_{i_{K+2}=j_{K+2}}^{j_{K+1}-N_{i}} B_{i_{K+2}}^{[i-1]} \cdots \bigotimes_{i_{K j}=j_{K j}}^{j_{K j-1}-N_{[i+2]}} B_{i_{K j}}^{[i+1]} \bigotimes_{l=1}^{j_{K j}-N_{[i+1]}} B_{l}^{i}\right) \\
& =\bigotimes_{k=1}^{n} B_{k}^{i} \oplus \bigoplus_{j=1}^{\infty} \bigoplus_{j_{1}=N j-\left(\sum_{k=1}^{K-1} N_{[i-k]}-1\right)}^{n} \bigoplus_{j_{2}=N j-\left(\sum_{k=1}^{K} N_{[i-k]}-1\right)}^{j_{1}-N_{i}} \\
& j_{2}-N_{[i-1]} \quad j_{3}-N_{[i-2]} \\
& \bigoplus_{j_{3}=N j-\left(2 N_{[i-1]}+\sum_{k=2}^{K} N_{[i-k]}-1\right)} \bigoplus_{j_{4}=N j-\left(2 \sum_{k=1}^{2} N_{[i-k]}+\sum_{k=3}^{K} N_{[i-k]}-1\right)} \\
& \ldots \bigoplus_{j_{K+2}=N j-\left(2 \sum_{k=1}^{K} N_{[i-k]}-1\right)}^{j_{K+1}-N_{i}} \bigoplus_{j_{K+3}=N j-\left(3 N_{[i-1]}+2 \sum_{k=2}^{K} N_{[i-k]}-1\right)}^{j_{K+2}-N_{[i-1]}} \ldots \\
& \ldots \bigoplus_{j_{2 K+2}=N j-\left(3 \sum_{k=1}^{K} N_{[i-k]}-1\right)}^{j_{2 K+1}-N_{i}} \cdots \bigoplus_{j_{K j}=1}^{j_{K j-1}-N_{[i+2]}}\left(\bigotimes_{i_{1}=j_{1}}^{n} B_{i_{1}}^{i} \bigotimes_{i_{2}=j_{2}}^{j_{1}-N_{i}} B_{i_{2}}^{[i-1]}\right. \\
& \bigotimes_{i_{3}=j_{3}}^{j_{2}-N_{[i-1]}} B_{i_{3}}^{[i-2]} \bigotimes_{i_{4}=j_{4}}^{j_{3}-N_{[i-2]}} B_{i_{4}}^{[i-3]} \cdots \bigotimes_{i_{K}=j_{K}}^{j_{K-1}-N_{[i+2]}} B_{i_{K}}^{[i+1]} \\
& \left.\bigotimes_{i_{K+1}=j_{K+1}}^{j_{K}-N_{[i+1]}} B_{i_{K+1}}^{i} \ldots \bigotimes_{i_{K j}=j_{K j}}^{j_{K j-1}-N_{[i+2]}} B_{i_{K j}}^{[i+1]} \bigotimes_{l=1}^{j_{K j}-N_{[i+1]}} B_{l}^{i}\right)
\end{aligned}
$$

where we used (4), the induction hypothesis and the representation in (11) for $X_{n-N_{i}}^{[i-1]}$ to obtain the first equality, we relabeled the indeces to obtain the second equality, and the last equality follows by putting all terms together. On the other hand, if $i$ is such that $N_{i}=0$ and $N_{[i-1]}>0$, then the expression for $X_{n}^{i}$ can be obtained in the same way since we now have an expression for $X_{n}^{i}$ for all $i$ with $N_{i}>0$, and thus in particular for $X_{n}^{[i-1]}$. Continuing in this fashion one can obtain $X_{n}^{i}$ for $i$ with $N_{i}=N_{[i-1]}=\cdots=N_{[i-(k-1)]}=0$ and $N_{[i-k]}>0$, for any $k=1, \cdots, K-1$.

\section{Cycle Times}

Recall that $C_{n}^{i}$ denotes the $n^{\text {th }}$ cycle time at station $i(i=1, \ldots, K)$. By a cycle time we mean the time between two successive departures of the same customer from a given station. Thus, the $n^{\text {th }}$ cycle time at station $i$ is computed as

$$
C_{n}^{i}=X_{N+n}^{i}-X_{n}^{i}
$$


Proposition 4.1 For $i \in\{1, \ldots, K\}$ and $n \geq N-N_{[i+1]}$, we have

$$
\mathbb{P}\left(C_{n}^{i}>x\right) \sim N \sum_{k=1}^{K} c_{k} \bar{F}(x) \quad \text { as } x \rightarrow \infty .
$$

Proof We first derive a lower bound for $C_{n}^{i}$. By summing up all service times that appear in the expression of $X_{n}^{i}$, we get

$$
\begin{aligned}
& X_{n}^{i} \leq B_{n}^{i} \ldots B_{1}^{i} \otimes B_{n-N_{i}}^{[i-1]} \ldots B_{1}^{[i-1]} \\
& \otimes B_{n-N_{i}-N_{[i-1]}}^{[i-2]} \ldots B_{1}^{[i-2]} \otimes \ldots \otimes B_{n-\left(N-N_{[i+1]}\right.}^{[i-K+1]} \ldots B_{1}^{[i-K+1]} .
\end{aligned}
$$

Note that $X_{n}^{i}$ is the maximum of sums of service times. In order to get a lower bound for $X_{n}^{i}$, for each $k, k=1, \ldots, K$, we pick that sum of service times which contains a maximal number of service times with upper index $k$. Each of these sums is bounded from below by considering only the service times with upper index $k$ and dropping all the rest. This yields

$$
\begin{aligned}
& X_{n}^{i} \geq B_{n}^{i} \ldots B_{1}^{i} \oplus B_{n-N_{i}}^{[i-1]} \ldots B_{1}^{[i-1]} \oplus B_{n-N_{i}-N_{[i-1]}}^{[i-2]} \ldots B_{1}^{[i-2]} \\
& \oplus B_{n-N_{i}-N_{[i-1]}-N_{[i-2]}}^{[i-3]} \ldots B_{1}^{[i-3]} \oplus \ldots \oplus B_{n-\left(N-N_{[i+1]}\right)}^{[i-K+1]} \ldots B_{1}^{[i-K+1]} .
\end{aligned}
$$

From (13) and (14),

$$
\begin{aligned}
& C_{n}^{i}=X_{N+n}^{i}-X_{n}^{i} \\
& \geq\left(B_{N+n}^{i} \ldots B_{n+1}^{i}-\left(B_{n-N_{i}}^{[i-1]} \ldots B_{1}^{[i-1]} \otimes B_{n-N_{i}-N_{[i-1]}}^{[i-2]} \ldots B_{1}^{[i-2]}\right.\right. \\
& \left.\left.\otimes \ldots \otimes B_{n-\left(N-N_{[i+1]}\right]}^{[i-K+1]} \ldots B_{1}^{[i-K+1]}\right)\right) \\
& \oplus\left(B_{N+n-N_{i}}^{[i-1]} \ldots B_{n+1-N_{i}}^{[i-1]}-\left(B_{n}^{i} \ldots B_{1}^{i} \otimes B_{n-N_{i}-N_{[i-1]}}^{[i-2]} \ldots B_{1}^{[i-2]}\right.\right. \\
& \left.\left.\otimes \ldots \otimes B_{n-\left(N-N_{[i+1]}\right.}^{[i-K+1]} \ldots B_{1}^{[i-K+1]}\right)\right) \\
& \oplus\left(B_{N+n-N_{i}-N_{[i-1]}}^{[i-2]} \cdots B_{n+1-N_{i}-N_{[i-1]}}^{[i-2]}\right. \\
& -\left(B_{n}^{i} \ldots B_{1}^{i} \otimes B_{n-N_{i}}^{[i-1]} \ldots B_{1}^{[i-1]} \otimes B_{n-N_{i}-N_{[i-1]}-N_{[i-2]}}^{[i-3]} \ldots B_{1}^{[i-3]}\right. \\
& \left.\left.\otimes \ldots \otimes B_{n-\left(N-N_{[i+1]}\right]}^{[i-K+1]} \ldots B_{1}^{[i-K+1]}\right)\right) \\
& \oplus \ldots \oplus\left(B_{N+n-\left(N-N_{[i+1]}\right.}^{[i-K+1]} \cdots B_{n+1-\left(N-N_{[i+1]}\right)}^{[i-K+1]}\right. \\
& \left.-\left(B_{n}^{i} \ldots B_{1}^{i} \otimes B_{n-N_{i}}^{[i-1]} \ldots B_{1}^{[i-1]} \otimes \ldots \otimes B_{n-\left(N-N_{[i+1]}-N_{[i+2]}\right)}^{[i-K+2]} \ldots B_{1}^{[i-K+2]}\right)\right) \\
& \geq\left(B_{N+n}^{i} \ldots B_{n+1}^{i} \oplus B_{n+N-N_{i}}^{[i-1]} \ldots B_{n+1-N_{i}}^{[i-1]}\right.
\end{aligned}
$$




$$
\begin{array}{r}
\left.\oplus \ldots \oplus B_{n+N-\left(N-N_{[i+1]}\right)}^{[i-K+1]} \ldots B_{n+1-\left(N-N_{[i+1]}\right)}^{[i-K+1]}\right) \\
-\left(B_{n}^{i} \ldots B_{1}^{i} \otimes B_{n-N_{i}}^{[i-1]} \ldots B_{1}^{[i-1]} \otimes \ldots \otimes B_{n-\left(N-N_{[i+1]}\right)}^{[i-K+1]} \ldots B_{1}^{[i-K+1]}\right) .
\end{array}
$$

Therefore, by Lemmas 8.4, 8.5 and 8.6

$$
\liminf _{x \rightarrow \infty} \frac{\mathbb{P}\left(C_{n}^{i}>x\right)}{\bar{F}(x)} \geq N \sum_{k=1}^{K} c_{k}
$$

We now obtain an upper bound on $C_{n}^{i}$. Note that it follows from (8) that $X_{n+N}^{i}$ can be expressed in the following form

$$
X_{n+N}^{i}=\bigoplus_{k=1}^{\kappa} \beta_{k}
$$

where $\beta_{k}$ is the sum of various combinations of service times that belong to the set

$\Theta=\left\{B_{1}^{i}, \ldots, B_{n+N}^{i}, B_{1}^{[i-1]}, \ldots, B_{n+N-N_{i}}^{[i-1]}, B_{1}^{[i-2]}, \ldots, B_{n+N-N_{i}-N_{i-1}}^{[i-2]}, \ldots, B_{1}^{[i-K+1]}, \ldots, B_{n+N-\left(N-N_{[i+1]}\right)}^{[i-K+1]}\right\}$

and $\kappa$ is an integer determined by $\max \left\{j: N j-\left(\sum_{k=1}^{K-1} N_{[i-k]}-1\right) \leq n+N\right\}$. Similarly, $X_{n}^{i}$ can be expressed as

$$
X_{n}^{i}=\bigoplus_{l=1}^{\eta} \gamma_{l}
$$

where $\gamma_{l}$ is the sum of various combinations of service times that belong to the set

$$
\Upsilon=\left\{B_{1}^{i}, \ldots, B_{n}^{i}, B_{1}^{[i-1]}, \ldots, B_{n-N_{i}}^{[i-1]}, B_{1}^{[i-2]}, \ldots, B_{n-N_{i}-N_{i-1}}^{[i-2]}, \ldots, B_{1}^{[i-K+1]}, \ldots, B_{n-\left(N-N_{[i+1]}\right)}^{[i-K+1]}\right\}
$$

and $\eta \leq \kappa$ is an integer determined by $\max \left\{j: N j-\left(\sum_{k=1}^{K-1} N_{[i-k]}-1\right) \leq n\right\}$. Consider $\beta_{k} \in$ $\left\{\beta_{1}, \ldots, \beta_{\kappa}\right\}$. We know that $\beta_{k}$ is written as a sum of the elements of some $\Upsilon_{k} \subset \Upsilon$ and some $\Theta_{k} \subset \Theta \backslash \Upsilon$. However, it then follows from (8) that there exists a $\gamma_{(k)} \in\left\{\gamma_{1}, \ldots, \gamma_{\eta}\right\}$ which also contains all the elements of $\Upsilon_{k}$. Then

$$
X_{n+N}^{i}-X_{n}^{i} \leq \bigoplus_{k=1}^{\kappa}\left(\beta_{k}-\gamma_{(k)}\right)
$$

where $\gamma_{(k)}, k=1, \ldots, \kappa$, is defined as above. Since for $k=1, \ldots, \kappa$

$$
\beta_{k}-\gamma_{(k)} \leq \bigotimes_{j=1}^{N} B_{n+j}^{i} \bigotimes_{j=1}^{N} B_{n+j-N_{i}}^{[i-1]} \ldots \bigotimes_{j=1}^{N} B_{n+j-\left(N-N_{[i-1]}\right)}^{[i-K+1]},
$$


Thus, from Lemma 8.6,

$$
C_{n}^{i} \leq \bigotimes_{j=1}^{N} B_{n+j}^{i} \bigotimes_{j=1}^{N} B_{n+j-N_{i}}^{[i-1]} \ldots \bigotimes_{j=1}^{N} B_{n+j-\left(N-N_{[i-1]}\right.}^{[i-K+1]} .
$$

$$
\limsup _{x \rightarrow \infty} \frac{\mathbb{P}\left(C_{n}^{i}>x\right)}{\bar{F}(x)} \leq N \sum_{k=1}^{K} c_{k}
$$

which together with (15) completes the proof.

Remark 4.1 The cycle time asymptotics is the same for each station. Thus, it does not matter at which station a cycle starts. This is different for the cycle time distribution, for which indeed one gets different results depending on where the cycle starts; see [8].

Remark 4.2 Let $c_{i_{0}}>0$ and $c_{i}=0$ for all $i \neq i_{0}$. Then

$$
\mathbb{P}\left(C_{n}^{i}>x\right) \sim N c_{i_{0}} \bar{F}(x) \sim N \bar{B}_{i_{0}}(x) \text { as } x \rightarrow \infty
$$

Thus the asymptotics depends only on the service time distribution with the heaviest tail.

\section{$5 \quad$ Waiting Times}

Let $W_{n}^{i}$ denote the $n^{\text {th }}$ waiting time at station $i(i=1, \ldots, K)$. Thus, $W_{n}^{i}$ is the time from the arrival of the $n^{\text {th }}$ customer at station $i$ to the beginning of his service at this station and it is computed as

$$
W_{n}^{i}=\left(X_{n-1}^{i}-X_{n-N_{i}}^{[i-1]}\right) \oplus 0
$$

with the convention that $X_{n}^{i}=0$ for all $n \leq 0$ and for all $i=1, \ldots, K$.

Proposition 5.1 For $i \in\{1, \ldots, K\}$ with $B_{i} \in \mathcal{S}$ and $n \geq N$, we have

$$
\mathbb{P}\left(W_{n}^{i}>x\right) \sim(N-1) \overline{B_{i}}(x) \sim(N-1) c_{i} \bar{F}(x) \quad \text { as } x \rightarrow \infty
$$

Proof Using the bounds on departure times given in (13) and (14), for $n \geq N$ we have

$$
\begin{gathered}
W_{n}^{i} \geq\left(B_{n-1}^{i} \ldots B_{1}^{i}-\left(B_{n-N_{i}}^{[i-1]} \ldots B_{1}^{[i-1]} \otimes B_{n-N_{i}-N_{[i-1]}}^{[i-2]} \ldots B_{1}^{[i-2]}\right.\right. \\
\left.\left.\otimes \ldots \otimes B_{n-\left(N-N_{[i+1]}\right)}^{[i-K+1]} \ldots B_{1}^{[i-K+1]} \otimes B_{n-N}^{i} \ldots B_{1}^{i}\right)\right) \oplus 0 \\
\geq B_{n-1}^{i} \ldots B_{n-N+1}^{i}-\left(B_{n-N_{i}}^{[i-1]} \ldots B_{1}^{[i-1]} \otimes B_{n-N_{i}-N_{[i-1]}}^{[i-2]} \ldots B_{1}^{[i-2]}\right. \\
\left.\otimes \ldots \otimes B_{n-\left(N-N_{[i-1]}\right)}^{[i-K+1]} \ldots B_{1}^{[i-K+1]}\right)
\end{gathered}
$$


and employing Lemma 8.4 and Lemma 8.6 we obtain

$$
\liminf _{x \rightarrow \infty} \frac{\mathbb{P}\left(W_{n}^{i}>x\right)}{\overline{B_{i}}(x)} \geq(N-1) .
$$

For an upper bound, we proceed in the same way as for the cycle times. Since $X_{n-1}^{i}$ is expressed as the maximum of sums of service times that belong to the set

$$
\left\{B_{1}^{i}, \ldots, B_{n-1}^{i}, B_{1}^{[i-1]}, \ldots, B_{n-1-N_{i}}^{[i-1]}, B_{1}^{[i-2]}, \ldots, B_{n-1-N_{i}-N_{[i-1]}}^{[i-2]}, \ldots, B_{1}^{[i-K+1]}, \ldots, B_{n-1-\left(N-N_{[i+1]}\right.}^{[i-K+1]}\right\}
$$

and $X_{n-N_{i}}^{[i-1]}$ is expressed as the maximum of sums of service times that belong to the set

$$
\left\{B_{1}^{[i-1]}, \ldots, B_{n-N_{i}}^{[i-1]}, B_{1}^{[i-2]}, \ldots, B_{n-N_{i}-N_{[i-1]}}^{[i-2]}, B_{1}^{[i-3]}, \ldots, B_{n-N_{i}-N_{[i-1]}-N_{[i-2]}}^{[i-3]}, \ldots, B_{1}^{[i-K]}, \ldots, B_{n-N}^{[i-K]}\right\}
$$

employing the arguments used in the derivation of the upper bound for cycle times we conclude that

$$
W_{n}^{i} \leq \bigotimes_{j=1}^{N-1} B_{n-j}^{i}
$$

Thus,

$$
\limsup _{x \rightarrow \infty} \frac{\mathbb{P}\left(W_{n}^{i}>x\right)}{\overline{B_{i}}(x)} \leq(N-1) .
$$

Since $\overline{B_{i}}(x) \sim c_{i} \bar{F}(x)$ as $x \rightarrow \infty$, the assertion follows.

\section{Residual Service Times}

In this section we analyze the asymptotic tail behaviour of the distribution of a residual service seen by an arriving customer. In contrast to an open system where the arrival process is independent of the service process, such a residual service time $B^{i \text {,res }}$ does not follow the residual service time distribution $B_{i}^{\mathrm{S}}(x)=\int_{0}^{x} \bar{B}_{i}(y) \mathrm{d} y / \beta_{i}$ where $\beta_{i}$ is the mean of $B_{i}(\cdot)$, but, up to a constant, has the same tail behaviour as $B_{i}(\cdot)$ given that the latter distribution is subexponential. Let $B_{n, k}^{i, \text { res }}$ denote the residual service time seen by the $n^{\text {th }}$ arriving customer at station $i, i=1, \ldots, K$, given that he sees $k$ customers in front of him. Then

$$
B_{n, k}^{i, \text { res }}=0 \oplus\left(X_{n-k+N_{i}}^{i}-X_{n}^{[i-1]}\right)
$$

with the convention that $X_{n}^{i}=0$ for all $n \leq 0$ and for all $i=1, \ldots, K$. 
Proposition 6.1 For $i \in\{1, \ldots K\}$ with $B_{i} \in \mathcal{S}, 1 \leq k \leq N-1$, and $n \geq N-N_{i}$,

$$
\mathbb{P}\left(B_{n, k}^{i, \text { res }}>x\right) \sim(N-k) \overline{B_{i}}(x) \sim(N-k) c_{i} \bar{F}(x) \quad \text { as } x \rightarrow \infty .
$$

Proof From the bounds in (13) and (14), for $n \geq N-N_{i}$

$$
\begin{aligned}
& B_{n, k}^{i, \text { res }} \\
& \geq B_{n-k+N_{i}}^{i} \ldots B_{1}^{i}-\left(B_{n}^{[i-1]} \ldots B_{1}^{[i-1]} \otimes \ldots \otimes B_{n-\left(N-N_{i}\right)}^{i} \ldots B_{1}^{i}\right) \\
& =B_{n-k+N_{i}}^{i} \ldots B_{n-\left(N-N_{i}\right)+1}^{i} \\
& \quad-\left(B_{n}^{[i-1]} \ldots B_{1}^{[i-1]} \otimes \ldots \otimes B_{n-\left(N-N_{[i-K+1]}-N_{[i-K]}\right)}^{[i-K+1]} B_{1}^{[i-K+1]}\right),
\end{aligned}
$$

and thus from Lemmas 8.4 and 8.6

$$
\liminf _{x \rightarrow \infty} \frac{\mathbb{P}\left(B_{n, k}^{i, \text { res }}>x\right)}{\overline{B_{i}}(x)} \geq(N-k) .
$$

To get an upper bound, we again proceed in the same way as for the cycle times. Note that $X_{n-k+N_{i}}^{i}$ is written as the maximum of sums of service times that belong to the set $\left\{B_{1}^{i}, \ldots, B_{n-k+N_{i}}^{i}, B_{1}^{[i-1]}, \ldots, B_{n-k}^{[i-1]}, B_{1}^{[i-2]}, \ldots, B_{n-k-N_{[i-1]}}^{[i-2]}, \ldots, B_{1}^{[i-K+1]}, \ldots, B_{n-k-\left(N-N_{i}-N_{[i+1]}\right)}^{[i-K+1]}\right\}$ and $X_{n}^{[i-1]}$ is written as the maximum of sums of service times that belong to the set $\left\{B_{1}^{[i-1]}, \ldots, B_{n}^{[i-1]}, B_{1}^{[i-2]}, \ldots, B_{n-N_{[i-1]}}^{[i-2]}, B_{1}^{[i-3]}, \ldots, B_{n-N_{[i-1]}-N_{[i-2]}}^{[i-3]}, \ldots, B_{1}^{[i-K]}, \ldots, B_{n-\left(N-N_{i}\right)}^{[i-K]}\right\}$

Employing the arguments used in the derivation of the upper bound for cycle times yields

$$
B_{n, k}^{i, \text { res }} \leq \bigotimes_{j=k}^{N-1} B_{n+N_{i}-j}^{i} .
$$

Therefore, from Lemma 8.6

$$
\limsup _{x \rightarrow \infty} \frac{\mathbb{P}\left(B_{n, k}^{i, \text { res }}>x\right)}{\overline{B_{i}}(x)} \leq(N-k)
$$

Again, since $\overline{B_{i}}(x) \sim c_{i} \bar{F}(x)$, this completes the proof.

Notice that the asymptotic constant in (17) is decreasing with respect to the number of customers an arriving customer sees in front of him. Thus, an intuitive explanation for the result of Proposition 6.1 could be as follows: the more customers there are waiting at a queue, the more 
likely it is that the ongoing service has started quite a while ago giving rise to the build up of a long queue, and is thus more likely to be finished soon. Similarly, seeing a short queue may indicate that the ongoing service has started not too long ago and therefore is more likely to have a longer residual service time.

Remark 6.1 Proposition 6.1 provides an alternative way to derive upper bounds for cycle and waiting times. Consider the worst case scenario, namely that a customer always sees $N-1$ customers in front of him when arriving at a station, with one service going on. Then,

$$
C_{n}^{i} \leq \sum_{k=1}^{K}\left(\sum_{j=1}^{N-1} B_{j}^{[i+k]}+B_{n, N-1}^{[i+k], \text { res }}\right),
$$

and thus

$$
\limsup _{x \rightarrow \infty} \frac{\mathbb{P}\left(C_{n}^{i}>x\right)}{\bar{F}(x)} \leq N \sum_{k=1}^{K} c_{k} .
$$

Similarly,

$$
\limsup _{x \rightarrow \infty} \frac{\mathbb{P}\left(W_{n}^{i}>x\right)}{\bar{F}(x)} \leq(N-1) c_{i}
$$

Note that these upper bounds agree with those obtained in the proofs of Propositions 4.1 and 5.1.

\section{$7 \quad$ Stationary Results}

In this section we assume that all service time distributions $B_{i}(\cdot), 1 \leq i \leq K$, have infinite support. Note that this assumption is always fulfilled for subexponential distributions. We observe the state of the cyclic network at departure epochs from station $i$. Let

$$
\mathbf{Y}^{i}(n)=\left(Y_{1}^{i}(n), \ldots, Y_{K}^{i}(n), Y_{K+1}^{i}(n), \ldots, Y_{2 K}^{i}(n)\right)
$$

be the system state vector, where $Y_{\ell}^{i}(n)$ and $Y_{K+\ell}^{i}(n)(\ell=1, \ldots, K)$ represent, respectively, the number of customers and the past service time of the ongoing service at station $\ell$ at the moment of the $n^{\text {th }}$ departure from station $i$. Hence, if $Y_{\ell}^{i}(n)=0$, then $Y_{K+\ell}^{i}(n)=0$. Moreover, let $\mathbf{Y}^{i}(0)=\left(N_{1}, \ldots, N_{K}, 0, \ldots, 0\right)$ which is consistent with the initial condition defined in Sections 1 and 2. Note that $\left\{\mathbf{Y}^{i}(n), n=0,1,2 \ldots\right\}$ is a Markov chain. Let $R=\left\{\mathbf{Y}^{0}\right\}$ be the one-point set such that $\mathbf{Y}^{0}$ is a $\{0,1, \ldots, N\}^{K} \times \mathbb{R}^{K}$ vector fulfilling

$$
Y_{i+1}^{0}=1, \quad Y_{i}^{0}=N-1, \quad Y_{\ell}^{0}=0 \quad(\ell \neq i, i+1), \quad Y_{K+\ell}^{0}=0 \quad(\ell=1, \ldots, K) .
$$


The event $R$ means that at a departure epoch from station $i$ the departing customer leaves all the other $N-1$ customers behind at station $i$. The one-step transition $\mathbf{Y}^{i}(n) \rightarrow \mathbf{Y}^{i}(n+1)=\mathbf{Y}^{0}$ takes place if and only if

$$
X_{n+1}^{i}>X_{n+N-N_{i}}^{[i-1]}
$$

Hence, the set $R$ is a regeneration set such that, for any initial marking,

$$
\mathbb{P}\left(\mathbf{Y}^{i}(n+1) \in R\right)=\mathbb{P}\left(X_{n+1}^{i}>X_{n+N-N_{i}}^{[i-1]}\right) \geq \mathbb{P}\left(B_{n+1}^{i}>X_{n+N-N_{i}}^{[i-1]}-X_{n+1-N_{i}}^{[i-1]}\right)
$$

where the last step follows from (4). Now, analogous to the proof of Proposition 4.1,

$$
X_{n+N-N_{i}}^{[i-1]}-X_{n+1-N_{i}}^{[i-1]} \leq \bigotimes_{j=1}^{N-1} B_{n+1-N_{i}+j}^{[i-1]} \bigotimes_{j=1}^{N-1} B_{n+1-N_{i}+j-N_{[i-1]}}^{[i-2]} \cdots \bigotimes_{j=1}^{N-1} B_{n+1-N_{i}+j-\left(N-N_{i}\right)}^{i},
$$

and therefore

$$
\begin{aligned}
& \mathbb{P}\left(X_{n+1}^{i}>X_{n+N-N_{i}}^{[i-1]}\right) \\
& \geq \mathbb{P}\left(B_{n+1}^{i}>\bigotimes_{j=1}^{N-1} B_{n+1-N_{i}+j}^{[i-1]} \bigotimes_{j=1}^{N-1} B_{n+1-N_{i}+j-N_{[i-1]}}^{[i-2]} \ldots \bigotimes_{j=1}^{N-1} B_{n+1-N_{i}+j-\left(N-N_{i}\right)}^{i}\right)>0 .
\end{aligned}
$$

Thus, $\mathbf{Y}^{i}(n)$ is a Harris ergodic Markov chain and has a unique stationary distribution. Namely, from (18) and Theorem 3.6 and Proposition 3.13 of [1] (see also $[2,3,6]$ ) we have the following theorem.

Theorem 7.1 If service time distributions at every station have infinite support, then there exists a random vector $\mathbf{Y}^{i}$ such that the distribution of $\mathbf{Y}^{i}(n)$ converges in total variation to the distribution of $\mathbf{Y}^{i}$. The Markov chain $\left\{\mathbf{Y}^{i}(n)\right\}$ couples with $\mathbf{Y}^{i}$ in finite time for any initial marking $N_{1}, N_{2}, \ldots, N_{K}$.

Note that the $n^{\text {th }}$ cycle at station $i, C_{n}^{i}=X_{N+n}^{i}-X_{n}^{i}$, can be expressed in terms of $\mathbf{Y}^{i}(n)$ and the completed services taking place in the time interval $\left[X_{n}^{i}, X_{n+N}^{i}\right)$. At the $k^{\text {th }}$ departure epoch from station $i$, the number of completed services since time 0 at the individual stations depends on the initial marking. Each station $l$ has completed at least $\max \left\{k-N_{i}, 0\right\}=\left(k-N_{i}\right)^{+} \geq(k-N)^{+}$and at most $k+N-N_{i}-1 \leq k+N-1$ services. We define the following sequence of random vectors for $n \geq N$

$$
\mathbf{B}^{\ell}(n)=\left(B_{n-N+1}^{\ell}, B_{n-N+2}^{\ell}, \ldots, B_{n+2 N-1}^{\ell}\right), \quad \ell=1,2, \ldots, K
$$


Since $\oplus$ and $\otimes$ are continuous operators, we have

$$
C_{n}^{i}=f_{1}\left(\mathbf{Y}^{i}(n), \mathbf{B}^{1}(n), \ldots, \mathbf{B}^{K}(n)\right)
$$

for $n \geq N$ and some continuous function $f_{1}:\{0,1, \ldots, N\}^{K} \times \mathbb{R}^{3 N K} \rightarrow \mathbb{R}_{+}$. Thus, since convergence in total variation implies weak convergence, from the continuous mapping theorem and Theorem 7.1 we obtain the following result.

Theorem 7.2 If service time distributions at each station have infinite support, then there exists a random variable $C^{i}=f_{1}\left(\mathbf{Y}^{i}, \mathbf{B}^{1}, \ldots, \mathbf{B}^{K}\right)$ such that $C_{n}^{i}$ converges in distribution to $C^{i}$ as $n \rightarrow \infty$. The sequence $C_{n}^{i}$ couples with $C^{i}$ in finite time for any initial marking $N_{1}, N_{2}, \ldots, N_{K}$.

It follows from Proposition 4.1 that for all $n \geq N-N_{[i+1]}$ and any $\varepsilon>0$ there exists $L$ such that for all $x>L$ we have

$$
(1-\varepsilon) N \sum_{k=1}^{K} c_{k} \bar{F}(x) \leq \mathbb{P}\left(C_{n}^{i}>x\right) \leq(1+\varepsilon) N \sum_{k=1}^{K} c_{k} \bar{F}(x),
$$

for $i \in\{1, \ldots, K\}$, and $c_{i}>0$. Taking limits as $n \rightarrow \infty$ and $\varepsilon \downarrow 0$ we obtain the following corollary.

Corollary 7.1 If service times at each station have infinite support, then for $i \in\{1, \ldots, K\}$ we have that

$$
\mathbb{P}\left(C^{i}>x\right) \sim N \sum_{k=1}^{K} c_{k} \bar{F}(x) \quad \text { as } x \rightarrow \infty .
$$

Note also that

$$
W_{n}^{i}=f_{2}\left(\mathbf{Y}^{i}(n), \mathbf{B}^{1}(n), \ldots, \mathbf{B}^{K}(n)\right)
$$

for some continuous function $f_{2}:\{0,1, \ldots, N\}^{K} \times \mathbb{R}^{3 N K} \rightarrow \mathbb{R}_{+}$. Thus, from Theorem 7.1 and Proposition 5.1 we can obtain the tail asymptotics of the distribution of the limiting waiting time at station $i\left(W^{i}\right)$.

Corollary 7.2 For $i \in\{1, \ldots K\}$ with $B_{i} \in \mathcal{S}$ we have

$$
\mathbb{P}\left(W^{i}>x\right) \sim(N-1) \overline{B_{i}}(x) \sim(N-1) c_{i} \bar{F}(x) \quad \text { as } x \rightarrow \infty .
$$

Similarly, we can obtain from Theorem 7.1 and Proposition 6.1 the asymptotics of the limiting residual distribution $Y_{K+i}^{[i-1]}$ given that $Y_{i}^{[i-1]}=k$, which we denote by $B_{k}^{i, \text { res }}$. 
Corollary 7.3 For $i \in\{1, \ldots K\}$ with $B_{i} \in \mathcal{S}, 1 \leq k \leq N-1$,

$$
\mathbb{P}\left(B_{k}^{i, \text { res }}>x\right) \sim(N-k) \overline{B_{i}}(x) \sim(N-k) c_{i} \bar{F}(x) \quad \text { as } x \rightarrow \infty
$$

\section{Appendix}

In this appendix we first give the proof of Lemma 3.1 and then recall some properties of the class $\mathcal{S}$ of subexponential distributions introduced in [13].

\subsection{Proof of Lemma 3.1}

First note that more explicitly (9) can be written as

$$
\begin{aligned}
& X_{n}^{i}=\bigoplus_{j=1}^{\infty} \underset{j_{1}=N j-\left(\sum_{k=1}^{K} N_{[i-k]}-1\right)}{n} \stackrel{\prod_{2}=N j-\left(2 N_{[i-K]}+\sum_{k=1}^{K-1} N_{[i-k]}-1\right)}{j_{1}-N_{i}} \\
& j_{2}-N_{[i-1]} \quad j_{3}-N_{[i-2]} \\
& \bigoplus \\
& j_{3}=N j-\left(2 N_{[i-K]}+2 N_{[i-1]}+\sum_{k=2}^{K-1} N_{[i-k]}-1\right) \quad j_{4}=N j-\left(2 N_{[i-K]}+2 \sum_{k=1}^{2} N_{[i-k]}+\sum_{k=3}^{K-1} N_{[i-k]}-1\right) \\
& j_{K+1}-N_{[i-K]} \quad j_{K+2}-N_{[i-1]}
\end{aligned}
$$

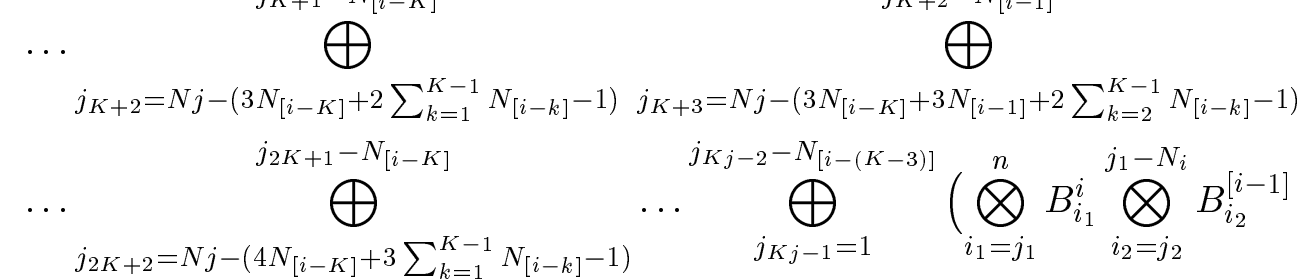

$$
\begin{aligned}
& \bigotimes_{i_{3}=j_{3}}^{j_{2}-N_{[i-1]}} B_{i_{3}}^{[i-2]} \bigotimes_{i_{4}=j_{4}}^{j_{3}-N_{[i-2]}} B_{i_{4}}^{[i-3]} \cdots \bigotimes_{i_{K}=j_{K}}^{j_{K-1}-N_{[i-(K-2)]}} B_{i_{K}}^{[i-K+1]} \\
& \left.\bigotimes_{i_{K+1}=j_{K+1}}^{j_{K}-N_{[i-(K-1)]}} B_{i_{K+1}}^{i} \cdots \bigotimes_{i_{K j-1}=j_{K j-1}}^{j_{K j-2}-N_{[i-(K-3)]}} B_{i_{K j-1}}^{[i-K+2]} \bigotimes_{l=1}^{j_{K j-1}-N_{[i-(K-2)]}} B_{l}^{[i-K+1]}\right) .
\end{aligned}
$$

Thus, we will show that (8) is equivalent to (19). Let

$$
\begin{aligned}
& j^{*}=\max \left\{j \in \mathbb{N}: N j-\left(\sum_{k=1}^{K-1} N_{[i-k]}-1\right) \leq n\right\}, \text { and } \\
& j_{0}^{*}=\max \left\{j \in \mathbb{N}: N j-\left(\sum_{k=1}^{K} N_{[i-k]}-1\right) \leq n\right\} .
\end{aligned}
$$

Note that $j_{0}^{*}$ always exists. However, if $n<N_{i}+1, j^{*}$ does not exist. We start with this case. Suppose $j^{*}$ does not exist which implies that $j_{0}^{*}=1$ and which is only possible if $N_{i}>0$. Then (8) 
reduces to $\bigotimes_{k=1}^{n} B_{k}^{i}$. On the other hand in (19) $j_{2}, j_{3}, \cdots, j_{K-1}$ vanish since

$$
j_{2} \leq j_{1}-N_{i}<1
$$

Moreover, the maximum in (19) is achieved for $j_{1}=1$ which yields $\bigotimes_{i_{1}=1}^{n} B_{i_{1}}^{i}$. For the rest of the proof we assume that $j^{*}$ exists, then in (8), $j=1, \cdots, j^{*}$ and in $(19), j=1, \cdots, j_{0}^{*}$. Note that $0 \leq j_{0}^{*}-j^{*} \leq 1$. Now suppose $N_{i}>0$ and $j^{*}=j_{0}^{*}$. We start with $\bigotimes_{k=1}^{n} B_{k}^{i}$ and $j=1$ term in (8),

$$
\begin{aligned}
& \bigotimes_{k=1}^{n} B_{k}^{i} \oplus \bigoplus_{j_{1}=N_{i}+1}^{n} \bigoplus_{j_{2}=1}^{j_{1}-N_{i}} \bigoplus_{j_{3}=1}^{j_{2}-N_{[i-1]}} \bigoplus_{j_{4}=1}^{j_{3}-N_{[i-2]}} \ldots \bigoplus_{j_{K}=1}^{j_{K-1}-N_{[i-(K-2)]}}\left(\bigotimes_{i_{1}=j_{1}}^{n} B_{i_{1}}^{i} \bigotimes_{i_{2}=j_{2}}^{j_{1}-N_{i}} B_{i_{2}}^{[i-1]} \ldots\right. \\
& \left.\bigotimes_{i_{K}=j_{K}}^{j_{K-1}-N_{[i-(K-2)]}} B_{i_{K}}^{[i-K+1]} \bigotimes_{l=1}^{j_{K}-N_{[i-(K-1)]}} B_{l}^{i}\right) \\
& =\bigoplus_{j_{1}=1}^{n} \bigoplus_{j_{2}=1}^{j_{1}-N_{i}} \cdots \bigoplus_{j_{K}=1}^{N_{[i-K+1]}}\left(\bigotimes_{i_{1}=j_{1}}^{n} B_{i_{1}}^{i} \bigotimes_{i_{2}=j_{2}}^{j_{1}-N_{i}} B_{i_{2}}^{[i-1]} \cdots \bigotimes_{i_{K}=1}^{j_{K-1}-N_{[i-(K-2)]}} B_{i_{K}}^{[i-K+1]}\right) \oplus \\
& \bigoplus_{j_{1}=N+1}^{n} \bigoplus_{j_{2}=N-N_{i}+1}^{j_{1}-N_{i}} \bigoplus_{j_{3}=N-N_{i}-N_{[i-1]}+1}^{j_{2}-N_{[i-1]}} \cdots \bigoplus_{j_{K}=N_{[i-K+1]}+1}^{j_{K-1}-N_{[i-(K-2)]}}\left(\bigotimes_{i_{1}=j_{1}}^{n} B_{i_{1}}^{i} \bigotimes_{i_{2}=j_{2}}^{j_{1}-N_{i}} B_{i_{2}}^{[i-1]} \ldots\right. \\
& \left.\cdots \bigotimes_{i_{K}=j_{K}}^{j_{K-1}-N_{[i-(K-2)]}} B_{i_{K}}^{[i-K+1]} \bigotimes_{l=1}^{j_{K}-N_{[i-(K-1)]}} B_{l}^{i}\right) .
\end{aligned}
$$

In order to obtain the above equality first note that for $j_{1}<N_{i}+1, j_{2}, j_{3}, \cdots, j_{K}$ vanish and for $j_{1}=1$ we obtain $\bigotimes_{k=1}^{n} B_{k}^{i}$ and for $2 \leq j_{1}<N_{i}+1$ we have

$$
\bigotimes_{i_{1}=j_{1}}^{n} B_{i_{1}}^{i} \leq \bigotimes_{k=1}^{n} B_{k}^{i}
$$

We then consider $j_{K} \leq N_{[i-K+1]}$ and $j_{K}>N_{[i-K+1]}$ separately to obtain (20) and (21). If $j_{K} \leq N_{[i-K+1]}, l$ vanishes and

$$
\bigotimes_{i_{K}=1}^{j_{K-1}-N_{[i-(K-2)]}} B_{i_{K}}^{[i-K+1]} \geq \bigotimes_{i_{K}=j_{K}}^{j_{K-1}-N_{[i-(K-2)]}} B_{i_{K}}^{[i-K+1]}
$$

for $1<j_{K}<N_{[i-K+1]}$. Finally, to obtain (21) for $j_{K} \geq N_{[i-K+1]}$, we need $j_{K-1} \geq N_{[i-K+1]}+$ $N_{[i-K+2]}+1, j_{K-2} \geq N_{[i-K+1]}+N_{[i-K+2]}+N_{[i-K+3]}+1, \cdots, j_{1} \geq N+1$. Note that (20) is the $j=1$ term in (19). We next consider $j=2$ term in (8) which is equal to

$$
\bigoplus_{j_{1}=N+N_{i}+1}^{n} \bigoplus_{j_{2}=N+1}^{j_{1}-N_{i}} \ldots \bigoplus_{j_{K}=N_{[i-K]}+N_{[i-K+1]}+1}^{j_{K-1}-N_{[i-K+2]}} \bigoplus_{j_{K+1}=N_{[i-K]}+1}^{j_{K}-N_{[i-K+1]}} \bigoplus_{j_{K+2}=1}^{j_{K+1}-N_{[i-K]}} \bigoplus_{j_{K+3}=1}^{j_{K+2}-N_{[i-1]}} \cdots \bigoplus_{j_{2 K}=1}^{j_{2 K-1}-N_{[i-K+}}
$$




$$
\begin{aligned}
& \left(\bigotimes_{i_{1}=j_{1}}^{n} B_{i_{1}}^{i} \bigotimes_{i_{2}=j_{2}}^{j_{1}-N_{i}} B_{i_{2}}^{[i-1]} \cdots \bigotimes_{i_{K}=j_{K}}^{j_{K-1}-N_{[i-K+2]}} B_{i_{2}}^{[i-1]} \bigotimes_{i_{K+1}=j_{K+1}}^{j_{K}-N_{[i-K+1]}} B_{i_{K+1}}^{[i-K]} \cdots\right. \\
& \left.\bigotimes_{i_{2 K}=j_{2 K}}^{j_{2 K-1}-N_{[i-K+2]}} B_{i_{2 K}}^{[i-K+1]} \bigotimes_{l=1}^{j_{2 K}-N_{[i-K+1]}} B_{l}^{i}\right)
\end{aligned}
$$

Putting (21) and (22) together yields

$$
\begin{aligned}
& \overbrace{}^{n} \quad j_{1}-N_{i} \quad j_{K-1}-N_{[i-K+2]} j_{K}-N_{[i-K+1]} j_{K+1}-N_{[i-K]} j_{K+2}-N_{[i-1]} \quad j_{2 K-1}-N_{[i-K+2]} \\
& \bigoplus_{j_{1}=N+1} \bigoplus_{j_{2}=N-N_{i}+1} \ldots \bigoplus_{j_{K}=N_{[i-K+1]}+1}^{\bigoplus} \bigoplus_{j_{K+1}=1} \bigoplus_{j_{K+2}=1} \bigoplus_{j_{K+3}=1} \ldots \bigoplus_{j_{2 K}=1} \\
& \left(\bigotimes_{i_{1}=j_{1}}^{n} B_{i_{1}}^{i} \bigotimes_{i_{2}=j_{2}}^{j_{1}-N_{i}} B_{i_{2}}^{[i-1]} \ldots \bigotimes_{i_{K}=j_{K}}^{j_{K-1}-N_{[i-K+2]}} B_{i_{2}}^{[i-1]} \bigotimes_{i_{K+1}=j_{K+1}}^{j_{K}-N_{[i-K+1]}} B_{i_{K+1}}^{[i-K]} \ldots\right. \\
& j_{2 K-1}-N_{[i-K+2]} j_{2 K}-N_{[i-K+1]} \\
& \left.\cdots \bigotimes_{i_{2 K}=j_{2 K}}^{j^{2}} B_{i_{2 K}}^{[i-K+1]} \bigotimes_{l=1}^{j^{2 K}} B_{l}^{i}\right) \\
& n \quad j_{1}-N_{i} \quad j_{K-1}-N_{[i-K+2]} \quad j_{K}-N_{[i-K+1]} j_{K+1}-N_{[i-K]} j_{K+2}-N_{[i-1]} \quad N_{[i-K+1]} \\
& =\bigoplus_{j_{1}=N+1}^{n} \bigoplus_{j_{2}=N-N_{i}+1}^{j_{1}-N_{i}} \cdots \bigoplus_{j_{K}=N_{[i-K+1]}+1}^{j_{K}} \bigoplus_{j_{K+1}=1}^{\bigoplus_{j_{K+2}=1}} \bigoplus_{j_{K+3}=1}^{\bigoplus_{[i-K+2]}} \cdots \bigoplus_{j_{2 K}=1}^{j_{K}-N_{[i-K+1]}} \\
& \left(\bigotimes_{i_{1}=j_{1}}^{n} B_{i_{1}}^{i} \bigotimes_{i_{2}=j_{2}}^{j_{1}-N_{i}} B_{i_{2}}^{[i-1]} \cdots \bigotimes_{i_{K}=j_{K}}^{j_{K-1}-N_{[i-K+2]}} B_{i_{2}}^{[i-1]} \bigotimes_{i_{K+1}=j_{K+1}}^{j_{K}-N_{[i-K+1]}} B_{i_{K+1}}^{[i-K]} \ldots\right. \\
& \left.\bigotimes_{i_{2 K}=1}^{j_{2 K-1}-N_{[i-K+2]}} B_{i_{2 K}}^{[i-K+1]}\right) \oplus \\
& \bigoplus_{j_{1}=2 N+1}^{n} \bigoplus_{j_{2}=2 N-N_{i}+1}^{j_{1}-N_{i}} \ldots \bigoplus_{j_{K}=N+N_{[i-K+1]}+1}^{j_{K-1}-N_{[i-K+2]}} \bigoplus_{j_{K+1}=N+1}^{j_{K}-N_{[i-K+1]}} \bigoplus_{j_{K+2}=N-N_{i}+1}^{j_{K+1}-N_{[i-K]}} \bigoplus_{j_{K+3}=N-N_{i}-N_{[i-1]}+1}^{j_{K+2}-N_{[i-1]}} \ldots \\
& \cdots \bigoplus_{j_{2 K-1}=N+N_{[i-K+1]}+N_{[i-K+2]} j_{2 K}=N_{[i-K+1]}+1}^{j_{2 K-1}-N_{[i-K+3]}}\left(\bigotimes_{i_{1}=j_{1}}^{j_{2 K-1}-N_{[i-K+2]}} B_{i_{1}}^{i_{1}} \bigotimes_{i_{2}=j_{2}}^{j_{1}-N_{i}} B_{i_{2}}^{[i-1]} \ldots \bigotimes_{i_{K}=j_{K}}^{j_{K-1}-N_{[i-K+2]}} B_{i_{2}}^{[i-1]}\right. \\
& \left.\bigotimes_{i_{K+1}=j_{K+1}}^{j_{K}-N_{[i-K+1]}} B_{i_{K+1}}^{[i-K]} \cdots \bigotimes_{i_{2 K}=1}^{j_{2 K-1}-N_{[i-K+2]}} B_{i_{2 K}}^{[i-K+1]} \bigotimes_{l=1}^{j_{2 K}-N_{[i-K+1]}} B_{l}^{i}\right)
\end{aligned}
$$

where the equality is obtained by considering $j_{2 K} \leq N_{[i-K+1]}$ and $j_{2 K}>N_{[i-K+1]}$ separately as is done above. Note that (23) is the $j=2$ term in (19). Continuing in this fashion for $j=j^{*}$ we have

$$
\begin{aligned}
& \overbrace{}^{n} \quad j_{1}-N_{i} \quad j_{K-1}-N_{[i-K+2]} \quad j_{K}-N_{[i-K+1]} \quad j_{K+1}-N_{[i-K]} \\
& \bigoplus_{j_{1}=N\left(j^{*}-1\right)+1} \bigoplus_{j_{2}=N\left(j^{*}-1\right)-N_{i}+1} \ldots \bigoplus_{j_{K}=N\left(j^{*}-2\right)+N_{[i-K+1]}+1} \bigoplus_{j_{K+1}=N\left(j^{*}-2\right)+1} \bigoplus_{j_{K+2}=N\left(j^{*}-2\right)-N_{i}+1} \ldots \\
& j_{2 K-1}-N_{[i-K+2]} \quad j_{2 K}-N_{[i-K+1]} \quad j_{K\left(j^{*}-1\right)}-N_{[i-K+1]} j_{K\left(j^{*}-1\right)+1}-N_{[i-K]} \quad j_{K j^{*}-1}-N_{[i-K+2]} \\
& \ldots \bigoplus_{j_{2 K}=N\left(j^{*}-3\right)+N_{[i-K+1]}+1} \bigoplus_{j_{2 K+1}=N\left(j^{*}-3\right)+1} \ldots \bigoplus_{j_{K\left(j^{*}-1\right)+1}=1} \bigoplus_{j_{K\left(j^{*}-1\right)+2}=1} \ldots \bigoplus_{j_{K j^{*}=1}} \\
& \left(\bigotimes_{i_{1}=j_{1}}^{n} B_{i_{1}}^{i} \bigotimes_{i_{2}=j_{2}}^{j_{1}-N_{i}} B_{i_{2}}^{[i-1]} \cdots \bigotimes_{i_{K}=j_{K}}^{j_{K-1}-N_{[i-K+2]}} B_{i_{2}}^{[i-1]} \bigotimes_{i_{K+1}=j_{K+1}}^{j_{K}-N_{[i-K+1]}} B_{i_{K+1}}^{[i-K]} \cdots\right.
\end{aligned}
$$




$$
\left.\ldots \bigotimes_{i_{j^{*} K}=j_{j^{*} K}}^{j_{j^{*} K-1}-N_{[i-K+2]}} B_{i_{j^{*} K}}^{[i-K+1]} \bigotimes_{l=1}^{j_{j^{*} K}-N_{[i-K+1]}} B_{l}^{i}\right) .
$$

In order to obtain (25) we combined the $j=j^{*}$ term of (8) with the remaining term from $j=j^{*}-1$ (i.e. the part corresponding to $\left.j_{\left(j^{*}-1\right) K}>N_{[i-K+1]}\right)$. Since $n<N j^{*}+1$,

$$
j_{j^{*} K} \leq n-\left(N j^{*}-N_{[i-K+1]}\right)<N_{[i-K+1]}+1 .
$$

Thus, $j_{j^{*} K}-N_{[i-K+1]}<1$ and the $l$ term vanishes in (25). Moreover, the maximum is achieved $j_{j^{*} K-1}-N_{[i-K+2]}$

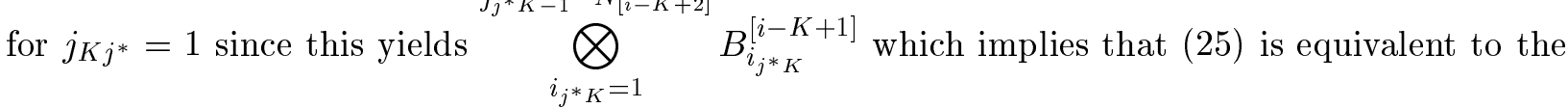
$j=j_{0}^{*}=j^{*}$ term in (19). This completes the proof for $N_{i}>0$ and $j^{*}=j_{0}^{*}$. If $N_{i}=0$ then clearly $j^{*}=j_{0}$. The proof of $N_{i}=0$ is the same as the one given above except in this case $\bigotimes_{k=1}^{n} B_{k}^{i}$ is redundant since for $j=1$ in (8), the expression corresponding to $j_{1}=1$ and $j_{2}=1$ dominates $\bigotimes_{k=1}^{n} B_{k}^{i}$. We next consider $j_{0}^{*}=j^{*}+1$ which is only possible if $N_{i}>0$. The proof will be the same as the proof of $j^{*}=j_{0}^{*}$ except for $j=j^{*}$ we now again need to split (25) into two parts by considering $j_{j^{*} K} \leq N_{[i-K+1]}$ and $j_{j^{*} K}>N_{[i-K+1]}$. Thus, (25) is written as

$$
\begin{aligned}
& \overbrace{}^{n} \quad j_{1}-N_{i} \quad j_{K-1}-N_{[i-K+2]} \quad j_{K}-N_{[i-K+1]} \quad j_{K+1}-N_{[i-K]} \\
& \bigoplus_{j_{1}=N\left(j^{*}-1\right)+1}^{n} \bigoplus_{j_{2}=N\left(j^{*}-1\right)-N_{i}+1} \ldots \bigoplus_{j_{K}=N\left(j^{*}-2\right)+N_{[i-K+1]}+1} \bigoplus_{j_{K+1}=N\left(j^{*}-2\right)+1} \bigoplus_{j_{K+2}=N\left(j^{*}-2\right)-N_{i}+1}^{j^{\prime}} \ldots \\
& j_{2 K-1}-N_{[i-K+2]} \quad j_{2 K}-N_{[i-K+1]} \quad j_{K\left(j^{*}-1\right)}-N_{[i-K+1]} j_{K\left(j^{*}-1\right)+1}-N_{[i-K]} \quad N_{[i-K+1]} \\
& \cdots \bigoplus_{j_{2 K}=N\left(j^{*}-3\right)+N_{[i-K+1]}+1} \bigoplus_{j_{2 K+1}=N\left(j^{*}-3\right)+1} \cdots \bigoplus_{j_{K\left(j^{*}-1\right)+1}=1} \bigoplus_{j_{K\left(j^{*}-1\right)+2}=1} \cdots \bigoplus_{j_{K j^{*}=1}} \\
& \left(\bigotimes_{i_{1}=j_{1}}^{n} B_{i_{1}}^{i} \bigotimes_{i_{2}=j_{2}}^{j_{1}-N_{i}} B_{i_{2}}^{[i-1]} \cdots \bigotimes_{i_{K}=j_{K}}^{j_{K-1}-N_{[i-K+2]}} B_{i_{2}}^{[i-1]} \bigotimes_{i_{K+1}=j_{K+1}}^{j_{K}-N_{[i-K+1]}} B_{i_{K+1}}^{[i-K]} \cdots\right. \\
& \left.\bigotimes_{i_{j^{*} K}=1}^{j_{j^{*} K-1}-N_{[i-K+2]}} B_{i_{j^{*} K}}^{[i-K+1]}\right) \oplus \\
& \bigoplus_{j_{1}=N j^{*}+1}^{n} \bigoplus_{j_{2}=N j^{*}-N_{i}+1}^{j_{1}-N_{i}} \ldots \bigoplus_{j_{K}=N\left(j^{*}-1\right)+N_{[i-K+1]}+1}^{j_{K-1}-N_{[i-K+2]}} \bigoplus_{j_{K+1}=N\left(j^{*}-1\right)+1}^{j_{K}-N_{[i-K+1]}} \bigoplus_{j_{K+2}=N\left(j^{*}-1\right)-N_{i}+1}^{j_{K+1}-N_{[i-K]}} \ldots \\
& j_{2 K-1}-N_{[i-K+2]} \quad j_{2 K}-N_{[i-K+1]} \quad j_{K\left(j^{*}-1\right)}-N_{[i-K+1]} \quad j_{K\left(j^{*}-1\right)+1}-N_{[i-K]} \\
& \cdots \bigoplus_{j_{2 K}=N\left(j^{*}-2\right)+N_{[i-K+1]}+1} \bigoplus_{j_{2 K+1}=N\left(j^{*}-2\right)+1}^{j_{2 K}} \cdots \bigoplus_{j_{K\left(j^{*}-1\right)+1}=N+1}^{j_{K\left(j^{*}-1\right)}} \bigoplus_{j_{K\left(j^{*}-1\right)+2}=N-N_{i}+1}^{j_{i i-K+1}} \cdots \\
& \bigoplus_{j_{K j^{*}}=N_{[i-K+1]}+1}^{j_{K j^{*}-1}-N_{[i-K+2]}}\left(\bigotimes_{i_{1}=j_{1}}^{n} B_{i_{1}}^{i} \bigotimes_{i_{2}=j_{2}}^{j_{1}-N_{i}} B_{i_{2}}^{[i-1]} \cdots \bigotimes_{i_{K}=j_{K}}^{j_{K-1}-N_{[i-K+2]}} B_{i_{2}}^{[i-1]} \bigotimes_{i_{K+1}=j_{K+1}}^{j_{K}-N_{[i-K+1]}} B_{i_{K+1}}^{[i-K]} \cdots\right. \\
& \left.\cdots \bigotimes_{i_{j^{*} K}=j_{j^{*} K}}^{j_{j^{*} K-1}-N_{[i-K+2]}} B_{i_{j^{*} K}}^{[i-K+1]} \bigotimes_{l=1}^{j_{j^{*} K}-N_{[i-K+1]}} B_{l}^{i}\right) .
\end{aligned}
$$


Note that (26) is the $j=j^{*}$ term in (19). On the other hand for $j=j_{0}^{*}=j^{*}+1$ in (19) we have

$$
\bigoplus_{j_{1}=N j^{*}+1}^{n} \bigoplus_{j_{2}=N j^{*}-N_{i}+1}^{j_{1}-N_{i}} \ldots \bigoplus_{j_{K}=N\left(j^{*}-1\right)+N_{[i-K+1]}+1}^{j_{j_{K}-1}-N_{[i-K+2]}} \bigoplus_{j_{K}-N_{[i-K+1}=N\left(j^{*}-1\right)+1}^{j_{j^{2}-1}-N_{[i-K+2]}} \bigoplus_{j_{K+2}=N\left(j^{*}-1\right)-N_{i}+1}^{j_{K}-N_{[i-K+1]}} \ldots
$$

The terms corresponding to $j_{K j^{*}+2}, j_{K j^{*}+3}, \cdots, j_{K j_{0}^{*}-1}$ disappear when $j=j_{0}^{*}$. Since $n<N j^{*}+$ $N_{i}+1$, we have

$$
j_{K j^{*}+2} \leq j_{K j^{*}+1}-N_{i}<n-N j^{*}-N_{i}<1
$$

Moreover, the maximum is achieved by setting $j_{K j^{*}+1}=1$ which implies that (28) is equivalent to (27). This completes the proof.

\subsection{Subexponential distributions}

As before, we write $f(x) \sim c g(x)$ to express that $\lim _{x \rightarrow \infty} f(x) / g(x)=c$ for two functions $f(x), g(x)$, and some constant $c \geq 0$.

Definition 8.1 A distribution function $F$ on $\mathbb{R}_{+}=[0, \infty)$ with $F(x)<1$ for all $x>0$ is called subexponential $(F \in \mathcal{S})$ if

$$
\overline{F^{* 2}}(x) \sim 2 \bar{F}(x)
$$

where $\bar{F}(x)=1-F(x)$ and $F^{* 2}$ denotes the convolution $F * F$.

The class $\mathcal{S}$ has some very useful properties. Those which are used in this paper are the following ones.

Lemma 8.1 Let $F, G$ be two distributions on $\mathbb{R}_{+}$and assume that there exists a constant $c \in(0, \infty)$ with $\bar{G}(x) \sim c \bar{F}(x)$. Then, $F \in \mathcal{S}$ if and only if $G \in \mathcal{S}$. 
Lemma 8.2 Let $F, G, H$ be distributions on $\mathbb{R}_{+}$such that $F \in \mathcal{S}, \bar{G}(x) \sim c_{1} \bar{F}(x)$ and $\bar{H}(x) \sim$ $c_{2} \bar{F}(x)$, where $c_{i} \in(0, \infty)$ for $i=1,2$. Then,

$$
\overline{G * H}(x) \sim\left(c_{1}+c_{2}\right) \bar{F}(x) .
$$

The results stated in Lemmas 8.1 and 8.2 are well-known. For a proof see for example [14] and $[17]$.

Lemma 8.3 Let $F \in \mathcal{S}$ and let $G$ be a distribution on $\mathbb{R}_{+}$such that $\bar{G}(x)=o(\bar{F}(x))$ as $x \rightarrow \infty$. Then $\overline{F * G}(x) \sim \bar{F}(x)$.

Lemma 8.4 Let $X$ and $Y \geq 0$ be independent random variables with distribution functions $F_{X} \in \mathcal{S}$ and $F_{Y}$, respectively. Then

$$
\mathbb{P}(X-Y>x) \sim \mathbb{P}(X>x) \text { as } x \rightarrow \infty .
$$

For the proofs of Lemmas 8.3 and 8.4, see [22]. We further need the following results, which are easily derived from the above lemmas.

Lemma 8.5 Let $F \in \mathcal{S}$ and let $G_{1}, \ldots, G_{n}, n \geq 1$, be distributions on $\mathbb{R}_{+}$such that $\bar{G}_{i}(x) \sim c_{i} \bar{F}(x)$ as $x \rightarrow \infty ; c_{i} \geq 0$. Then,

$$
1-\prod_{i=1}^{n} G_{i}(x) \sim \sum_{i=1}^{n} c_{i} \bar{F}(x) .
$$

Proof For $n=1$, the assertion is true. Now assume that (29) holds for some $n \geq 1$. Since

$$
1-\prod_{i=1}^{n+1} G_{i}(x)=\left(1-\prod_{i=1}^{n} G_{i}(x)\right) G_{n+1}(x)+1-G_{n+1}(x),
$$

the assumptions imply that

$$
\lim _{x \rightarrow \infty} \frac{1-\prod_{i=1}^{n+1} G_{i}(x)}{\bar{F}(x)}=\lim _{x \rightarrow \infty} \frac{1-\prod_{i=1}^{n} G_{i}(x)}{\bar{F}(x)} G_{n+1}(x)+\lim _{x \rightarrow \infty} \frac{\bar{G}_{n+1}(x)}{\bar{F}(x)}=\sum_{i=1}^{n} c_{i}+c_{n+1} .
$$

Lemma 8.6 Let $F \in \mathcal{S}$ and let $F_{1}, \ldots, F_{n}, n \geq 1$, and $G_{1}, \ldots, G_{m}, m \geq 1$, be distributions on $\mathbb{R}_{+}$ such that $\bar{F}_{i}(x) \sim c_{i} \bar{F}(x)$ with $c_{i}>0,1 \leq i \leq n$, and $\bar{G}_{i}(x)=o(\bar{F}(x))$ for $1 \leq i \leq m$. Then,

$$
\overline{F_{1} * \ldots * F_{n} * G_{1} * \ldots * G_{m}}(x) \sim \sum_{i=1}^{n} c_{i} \bar{F}(x) .
$$


Proof By induction it follows immediately from Lemma 8.2 that

$$
\overline{F_{1} * \ldots * F_{n}}(x) \sim \sum_{i=1}^{n} c_{i} \bar{F}(x) .
$$

Thus, by Lemma $8.1, H \stackrel{\text { def }}{=} F_{1} * \ldots * F_{n} \in \mathcal{S}$. Since, for $1 \leq i \leq m$,

$$
\lim _{x \rightarrow \infty} \frac{\bar{G}_{i}(x)}{\bar{H}(x)}=\lim _{x \rightarrow \infty} \frac{\bar{G}_{i}(x)}{\bar{F}(x)} \frac{\bar{F}(x)}{\bar{H}(x)}=0,
$$

(i.e., $\left.\bar{G}_{i}(x)=o(\bar{H}(x))\right)$ from Lemma 8.3 we have in particular $\overline{H * G_{1}}(x) \sim \bar{H}(x)$. Therefore the assertion is true for $m=1$, because $\bar{H}(x) \sim \sum_{i=1}^{n} c_{i} \bar{F}(x)$. Assume that the assertion holds for some $m \in \mathbb{N}$. Then,

$$
\lim _{x \rightarrow \infty} \frac{1-G_{m+1}(x)}{1-H * G_{1} * \ldots * G_{m}(x)}=\lim _{x \rightarrow \infty} \frac{1-G_{m+1}(x)}{\bar{H}(x)} \frac{\bar{H}(x)}{\sum_{i=1}^{n} c_{i} \bar{F}(x)} \frac{\sum_{i=1}^{n} c_{i} \bar{F}(x)}{1-H * G_{1} * \ldots * G_{m}(x)}=0,
$$

(i.e., $\left.\bar{G}_{m+1}(x)=o\left(\overline{H * G_{1} * \ldots * G_{m}}(x)\right)\right)$. Thus, by Lemma 8.3 and the induction hypothesis, the assertion follows.

\section{References}

[1] Asmussen, S. (1987) Applied Probability and Queues, John Wiley and Sons, New York.

[2] Baccelli, F. and Brémaud, P. (1992) Elements of Queueing Theory, Springer-Verlag, Berlin.

[3] Baccelli, F., Cohen, G., Olsder, G.J. and Quadrat, J.-P. (1992) Synchronization and Linearity: An Algebra for Discrete Event Systems, Springer Verlag and Sons, Chichester.

[4] Bacceli F., Schlegel S. and Schmidt V. (1999) "Asymptotics of stochastic networks with subexponential service times," Queueing Systems 33, 205-232.

[5] Baccelli, F., and Foss, S. (2001) "Moments and tails in monotone-separable stochastic networks," Rapport de Recherche de l'INRIA-Rocquencourt.

[6] Borovkov, A. (1984) Asymptotic Methods in Queueing Theory, John Wiley and Sons, New York. 
[7] Borovkov, A. (1986) "Limit theorems for service networks," Teoeriya Veroyatn. Primen., 31(3), 474-490.

[8] Boxma, O.J. (1983) "The cyclic queue with one general and one exponential server," Adv. Appl. Prob., 15, 857-873.

[9] Boxma, O.J. (1984) "Joint distribution of sojourn time and queue length in the M/G/1 queue with (in)finite capacity," European Journal of Operations Research 16, 246-256.

[10] Boxma, O.J. (1988) "Sojourn times in cyclic queues - the influence of the slowest server," Computer Performance and Reliability, 13-24, G. Iazeolla, P. Courtois and O. Boxma (eds.), North-Holland Publishing Co., Amsterdam.

[11] Boxma, O.J. and Donk, P. (1982) "On response time and cycle time distribution in a two-stage cyclic queue," Performance Evaluation, 2, 181-194.

[12] Boxma, O.J., Kelly, F.P. and Konheim, A.G. (1984) "The product form for sojourn time distributions in cyclic exponential queues," J.Assoc.Comput.Mach., 31, 128-133.

[13] Chistyakov, V.P. (1964) "A theorem on sums of independent, positive random variables and its applications to branching processes," Theory Prob. Appl. 9, 640-648.

[14] Cline, D.B.H. (1986) "Convolution tails, product tails and domains of attraction," Prob. Theory Rel. Fields, 72, 529-557.

[15] Chow, W.M. (1980) "The cycle time distribution of exponential cyclic queues," J.Assoc.Comput.Mach., 27, 281-286.

[16] Daduna, H. and Szekli, R. (1999) "Monotonicity and dependence properties of sojourn and cycle times in closed networks," Manuscript.

[17] Embrechts, P. and Goldie, C.M. (1982) "On convolution tails," Stoch. Proc. Appl., 13, 263-278.

[18] Gordon, W.J. and Newell, G.F. (1967) "Closed queueing networks with exponential servers," Operations Research, 15, 252-267. 
[19] Huang, T. and Sigman, K. (1999) "Steady state asymptotics for tandem, split-match and other feedforward queues with heavy tailed service," Queueing Systems, 33, 233-259.

[20] Klüppelberg, C. (1988) "Subexponential distributions and integrated tails," J. Appl. Probab., 25, $132-141$.

[21] Morozov, E. (1990) "Regeneration of a closed queueing network," Journal of Mathematical Sciences, 69(4), 1186-1192.

[22] Pitman, E.J.G. (1980) "Subexponential distribution functions," J. Austral. Math. Soc., (Series A), 29, 337-347.

[23] Resing, J.A.C., R.E. de Vries, G. Hooghiemstra, M.S. Keane and Olsder, G.J. (1990) "Asymptotic behaviour of random discrete event systems," Stoch. Proc. Appl., 36, 195-216.

[24] Schassberger, R. and Daduna, H. (1983) "The time for a round trip in a cycle of exponential queues," J.Assoc.Comput.Mach., 30, 146-150. 